\title{
MESURES ASSOCIEES AUX FONCTIONNELLES ADDITIVES DE MARKOV. I
}

\author{
PAR \\ D. $\operatorname{REVUZ}\left({ }^{1}\right)$
}

\begin{abstract}
With each additive functional of Markov processes we associate a measure and characterize, under duality hypotheses, those which correspond to $\sigma$-finite measures. This enables us to weaken the hypotheses of Meyer's theorem on representation of potentials of measures as potentials of additive functionals. We characterize also the measures which are associated with continuous additive functionals. This leads us to show that for each finite continuous additive functional of the process there exists a finite continuous additive functional of the dual process such that the corresponding time-changed processes are in duality. Similar results are also stated for subprocesses which generalize results by Hunt and Blumenthal and Getoor.
\end{abstract}

Sous l'hypothèse $(F)$ de Hunt, Meyer [10] a montré qu'il existe une correspondance bijective entre les fonctionnelles additives de potentiel fini et les mesures qui ne chargent pas les ensembles polaires et dont le potentiel est fini. Cette correspondance s'exprime par la relation:

$$
E_{x} \int_{0}^{\infty} e^{-\alpha t} d A_{t}=\int U^{\alpha}(x, y) \nu_{A}(d y)
$$

où $\nu_{A}$ est la mesure associée à la fonctionnelle $A$, et où $\alpha$ est un nombre réel positif.

D'autre part McKean et Tanaka [8], Volkonskiǐ [17] et [18] et Ventzel' [20] ont établi pour le mouvement brownien une correspondance plus générale entre les fonctionnelles finies et une large classe de mesure. Sous l'hypothèse (F), Blumenthal et Getoor ont dans [5] étendu ces résultats à une classe de fonctionnelles continues contenue dans la classe des fonctionnelles finies; la recherche des mesures associées se fait grâce aux théorèmes de Meyer.

Dans [1] et [2] au contraire nous avons montré que dans le cas récurrent on peut définir explicitement une mesure associée à toute fonctionnelle et nous avons donné des applications de cette définition à des théorèmes ergodiques. Le but de cet article est de montrer qu'on peut étendre cette définition au cas transient, et donner certaines applications de ces mesures sans hypothèse de dualité. Nous

Received by the editors June 14, 1969.

AMS Subject Classifications. Primary 6062.

Key Words and Phrases. Dual processes, reference excessive measure, $\sigma$-integrable functionals, accessible terminal times, natural potentials, regular potentials, random-time change, subprocesses.

${ }^{(1)}$ Equipe de Recherche $\mathrm{n}^{\circ} 1$ "Processus stochastiques et applications" dépendant de la Section $n^{\circ} 2$ "Théories Physiques et Probabilités" associée au C.N.R.S.

Copyright (C) 1970, American Mathematical Society 
essayerons ensuite de caractériser les fonctionnelles qui correspondent à des mesures $\sigma$-finies, nous trouverons ainsi une classe de fonctionnelles plus étendue que celle étudiée par Blumenthal et Getoor; elle contient notamment toutes les fonctionnelles finies pour lesquelles nous développerons les applications aux changements de temps données dans [5]; les caractérisations nous semblent plus simples que celles de [5]. Ceci sera fait sous l'hypothèse qu'il existe deux processus standards en dualité, mais sans supposer les résolvantes fortement felleriennes, c'est à dire une hypothèse une peu plus faible que (F), sous laquelle nous aurons aussi les théorèmes de Meyer.

Je tiens à remercier ici M. M. J. Neveu et P. A. Meyer dont l'enseignement et les conseils m'ont été si profitables, $\mathrm{M}$. Dixmier qui a bien voulu présider mon Jury et M. Guichardet qui m'a fourni le sujet de ma 2ème Thèse.

\section{Notations et preliminaires.}

I.1. Nous prendrons presque toujours les notations de [4], auquel nous renvoyons pour le définition de la plupart des termes employés ici. Nous considérons un processus standard

$$
X=\left\{\Omega, \mathscr{F},\left(\mathscr{F}_{t}\right)_{t \in \boldsymbol{R}_{+}},\left(X_{t}\right)_{t \in \boldsymbol{R}_{+}},\left(P_{x}\right)_{x \in E},\left(\theta_{t}\right)_{t \in \boldsymbol{R}_{+}}, \zeta\right\}
$$

à valeur dans un espace $(E, \mathscr{E})$ localement compact à base dénombrable. Nous ferons dans tout cet article l'hypothèse (indispensable dans le cas transient) qu'il existe une mesure de référence [4, p. 196], et nous étudierons simultanément les deux cas suivants: $1^{\circ} / E$ est une seule classe récurrente [1] et [2]; il existe donc alors une mesure invariante $\xi \sigma$-finie et équivalente aux mesures $U^{\alpha}(x, \cdot) ; 2^{\circ} / \mathrm{le}$ noyau de la théorie du potentiel est un noyau propre [15, p. 215]; si $m$ est une mesure de référence bornée, $\xi=m U(\cdot)$ est alors une mesure excessive $\sigma$-finie. Dans les deux cas $\xi$ est une mesure excessive de référence et c'est cette propriété qui nous servira essentiellement. Si dans l'énoncé d'un théorème il faut distinguer le cas récurrent du cas transient nous le ferons explicitement. Remarquons encore que si un processus est irréductible, on se trouve dans un des deux cas visés ci-dessus.

I.2. Notons encore que l'intégrale d'une fonction $f$ par rapport à une mesure $\mu$ sera notée indifférement:

$$
\int f d \mu=\langle\mu, f\rangle=\mu(f) .
$$

Nous désignerons par $b \mathscr{E}$ (resp. $b \mathscr{E}_{+}, b \mathscr{E}^{*}, b \mathscr{E}_{+}^{*}$ ) l'ensemble des fonctions boréliennes bornées (resp. boréliennes bornées positives, universellement mesurable bornée, ....).

Une relation qui a lieu $P_{x}$-presque sûrement pour tout $x$ de $E$, sera dite avoir lieu presque-sûrement et l'on écrira seulement p.s.

I.3. Une fonctionnelle additive $A$ de $X$, est une famille $\left(A_{t}\right)_{t \in R_{+}}$de variables aléatoires positives telle que: 
(1) L'application $t \rightarrow A_{t}(\omega)$ est p.s. continue à droite, nulle à l'instant 0 ;

(2) $A_{t}$ est $\mathscr{F}_{t}$-mesurable;

(3) Pour tout couple $(s, t)$ on a $A_{t+s}=A_{t}+A_{s} \circ \theta_{t}$ p.s.;

(4) Pour tout $t \geqq \zeta, A_{t}=A_{\zeta}$-p.s.

La fonctionnelle additive sera dite naturelle si les applications $t \rightarrow X_{t}$ et $t \rightarrow A_{t}$ n'ont presque-sùrement aucune discontinuité commune. Pour abréger nous écrirons FAC (resp. FAN) au lieu de fonctionnelle additive continue (resp. naturelle). Deux fonctionnelles $A$ et $B$ seront dites équivalentes si les fonctions $t \rightarrow A_{t}$ et $t \rightarrow B_{t}$ sont presque sûrement égales. Dans toute la suite l'égalité entre fonctionnelles voudra dire équivalence.

Si $A$ est naturelle et si $a, b$ sont deux nombres réels strictement positifs nous poserons $T_{b}^{a}(\omega)=\inf \left\{t ; a<A_{t}(\omega)-A_{\iota}-(\omega) \leqq b\right\}$ si cet ensemble n'est pas vide et $T_{b}^{a}(\omega)=\infty$ sinon; $T_{b}^{a}$ est un temps d'arrêt accessible; terminal et effilé en tout point (cf. [4], [6] et [12, Partie IV]). On appellera $T_{b}^{a(n)}$ les itérés de $T_{b}^{a}$, c'est à dire les temps d'arrêt définis par $T^{0}=0, T_{b}^{a(n)}=T_{b}^{a(n-1)}+T_{b}^{a} \circ \theta_{T_{b}^{a(n-1)}}$; l'expression

$$
\sum_{n=1}^{\infty} 1_{\left(T_{b}^{a(n)} \leqq t\right)}\left(A_{T_{b}^{a(n)}}-A_{\left.T_{b}^{a(n)-}\right)}\right)
$$

définit alors une FAN. Plus généralement si $\tau$ est un temps d'arrêt terminal et effilé en tout point, et si $\tau^{(n)}$ est son nième itéré l'expression

$$
B_{t}^{\tau}=\sum_{n=1}^{\infty} 1_{\left(\imath^{(n)} \leqq t, \tau^{(n)}<\zeta\right)}
$$

est une fonctionnelle additive, naturelle si $\tau$ est accessible [4, p. 172].

Nous appellerons $\Phi$ (resp. $\Phi_{c}$ ) la classe des fonctionnelles additives (resp. FAC) telles que $\{t<\zeta\} \subset\left\{A_{t}<\infty\right\}$. Si $f \in b \mathscr{E}_{+}^{*}$ et si $A \in \Phi$, l'expression

$$
B_{t}=\int_{0}^{t} f\left(X_{s}\right) d A_{s}
$$

définit une fonctionnelle additive de $X$ que l'on notera (fA) ou f.A. On sait qu'il n'en est pas forcément de même si l'on supprime l'une des deux hypothèses et il faut alors vérifier les différentes conditions dans chaque cas. Nous aurons ainsi à considérer les expressions $B_{t}$ pour $f \in b_{\mathscr{E}_{+}^{*}}^{*}$ et $A$ quelconque. Ce ne sont plus des fonctionnelles additives, mais les applications $t \rightarrow B_{t}$ sont p.s. croissantes et continues à droite et définissent donc des mesures sur $\boldsymbol{R}_{+}$; de plus il est facile de voir que

$$
B_{t+s} \leqq B_{t}+B_{s} \circ \theta_{t} \text { p.s.; }
$$

la relation d'additivité étant vérifiée si $\left\{B_{t}<\infty, t<\zeta\right\} \subset\left\{A_{t}<\infty\right\}$.

Pour toute fonction $f \in \mathscr{E}_{+}^{*}$, on définit le $\alpha$-potentiel de $f$ par rapport à $A$ comme l'expression

$$
U_{A}^{\alpha} f(x)=E_{x} \int_{0}^{\infty} e^{-\alpha t} f\left(X_{t}\right) d A_{t}
$$


sous certaines hypothèses ces potentiels vérifient l'équation résolvente

$$
U_{A}^{\alpha}-U_{A}^{\beta}=(\beta-\alpha) U^{\alpha} U_{A}^{\beta} ;
$$

sous l'hypothèse définie plus haut où $B_{t}$ n'est pas forcément une fonctionnelle additive on a encore si $\beta \geqq \alpha$,

$$
U_{A}^{\alpha} f(x)-U_{A}^{\beta} f(x) \leqq(\beta-\alpha) U^{\alpha} U_{A}^{\beta} f(x) .
$$

I.4. Lemme. Soit $A^{n}(t)$ une série de fonctions de $\boldsymbol{R}_{+} \cup\{0\}$ dans $\boldsymbol{R}_{+}$non décroissantes et continues (resp. continues à droite) de somme $A(t)$; si $A(t)$ n'est pas continue (resp. continue à droite) en un point $T$ la discontinuité est infinie, c'est à dire que $A(T+\varepsilon)-A(T)=\infty$ pour tout $\varepsilon>0$. Si les $A^{n}(t)$ sont continues, $A(t)$ est continue sur $[0, T]$.

Démonstration. Supposons $A(t)<\infty$, alors pour tout $s \leqq t$

$$
A(s)-\sum_{1}^{N} A^{n}(s)=\sum_{N+1}^{\infty} A^{n}(s) \leqq \sum_{N+1}^{\infty} A^{n}(t)
$$

puisque les $A^{n}$ sont croissantes, et cette dernière quantité tend vers zéro puisqu'on a supposé que $A(t)<\infty$, i.e. que la série $\sum_{1}^{\infty} A^{n}(t)$ converge. La série $\sum_{1}^{\infty} A^{n}(s)$ converge donc uniformément sur $[0, t]$ et sa somme est donc continue (resp. continue à droite) sur $[0, t]$. Par suite si $A_{t}$ admet une discontinuité en $T, A_{T+\varepsilon}=+\infty$ pour tout $\varepsilon>0$.

I.5. Nous allons tirer de ce lemme les conséquences suivantes très utiles dans la suite.

Proposition. Soit $A_{t}^{n}$ une série de FAC de somme $A_{t}$. Si $A_{t}$ admet une discontinuité à l'instant $T$, cette discontinuité est infinie et il existe un point $x$ de $E$ tel que $P_{x}(T=0)=1$. Dans le cas contraire $A_{t}$ est une FAC.

Démonstration. On a évidemment $A_{0}=0$ p.s., $A_{t}$ est $\mathscr{F}_{t}$-mesurable et pour tout couple de temps d'arrêt $A_{T+S}=A_{T}+A_{S}\left(\theta_{T}\right)$ p.s. D'après le lemme précédent si $A_{t}$ admet une discontinuité à l'instant $T$ cette discontinuité est unique et elle est infinie. $T$ est un temps d'arrêt; supposons que pour tout $x$ de $E, P_{x}(T>0)=1$. D'après le lemme précédent $A_{t}$ est continue sur [0,T] et donc $A_{t}\left(\theta_{T}\right) \operatorname{sur}\left[0, T\left(\theta_{T}\right)\right]$; comme $A_{T+t}=A_{T}+A_{t}\left(\theta_{T}\right)$ il en résulte que $A_{t}$ est aussi continue sur $\left[T, T+T \circ \theta_{T}\right]$; elle est donc continue en $T$ et par suite n'admet aucune discontinuité. S'il y a une discontinuité, il existe donc un point $x$ tel que $P_{x}[T=0]>0$ et d'après la loi du tout ou rien de Blumenthal $\left[4\right.$, p. 30] tel que $P_{x}[T=0]=1$.

Si $A_{t}$ est continue il est clair que c'est une FAC, mais remarquons toutefois qu'elle peut devenir infinie avant $\zeta$ même si les $A^{n}$ sont finies sur [0, $\zeta[$.

I.6. Proposition. Une FAN dont les sauts sont finis presque-sûrement peut se décomposer en la somme d'une FAC et d'une FAN purement discontinue.

Démonstration. Soit $\left(a_{n}\right)_{n \in Z}$ une suite croissante de nombres réels strictement 
positifs tels que $\lim _{n \rightarrow-\infty} a_{n}=0$ et $\lim _{n \rightarrow+\infty} a_{n}=+\infty$, et soit $T_{k}=T_{a_{k+1}}^{a_{k}}$ avec les notations de I.3. Par commodité nous pouvons rearranger ces temps d'arrêts en une suite $\left(\tau_{k}\right)_{k \in N}$ de temps d'arrêt qui par hypothèse sont tels que les $\tau_{k}^{(n)}(k \in N, n \in N)$ épuisent tous les sauts de $A_{t}$. Pour tout $K$,

$$
D_{t}^{k}=\sum_{k=1}^{K} \sum_{n=1}^{\infty} 1_{\left\{\tau_{k}^{(n)} \leqq t\right\}}\left(A_{\tau_{k}^{(n)}}-A_{\tau_{k}^{(n)}-}\right)
$$

est une FAN et $A_{t}-D_{t}^{K}$ est une FAN; ceci entraine que pour tout couple $(s, t)$ $A_{t}-A_{s} \geqq D_{t}^{K}-D_{s}^{K}$. Posons $D_{t}=\lim _{K \rightarrow \infty} D_{t}^{K} ; D_{t}$ ne peut avoir de discontinuité infinie car cela entrainerait que $A_{t}$ a une discontinuité infinie ce qui est exclu par l'hypothèse; d'après le Lemme I.4 $D_{t}$ est donc une FAN. Par passage à la limite monotone $A_{t}-D_{t}$ définit alors une fonctionnelle qui est continue par construction.

I.7. Dans ce paragraphe nous voudrions montrer que même pour les fonctionnelles qui ne sont pas dans $\Phi$ on peut définir des fonctionnelles traces sur les ensembles. Le lecteur qui ne s'intéresse qu'aux fonctionnelles finies pourra sauter ce paragraphe.

On sait ([4, p. 205], [7]) que sous l'hypothèse de continuité absolue toute FAC et toute fonctionelle de $\Phi$ est équivalente à une fonctionnelle parfaite et nous montrerons plus loin que le raisonnement de [7] s'étend à toutes les fonctionnelles à sauts finis.

Soit alors $A$ une fonctionnelle additive parfaite qui n'est pas dans $\Phi$ et $S=\inf \left\{t: A_{t}=\infty\right\}$; nous supposons de plus que les sauts de $A$ sont finis. Soit $f \in b \mathscr{E}_{+}$, on pose

$$
B_{t}=\int_{0}^{t} f\left(X_{s}\right) d A_{s}
$$

d'après ce qui précède, il existe un ensemble $\Lambda \in \mathscr{F}$, de probabilité nulle pour toute loi $P_{x}$, et tel que si $\omega \notin \Lambda$ et si $t+s \leqq S(\omega)$,

$$
B_{t+s}(\omega)=B_{t}(\omega)+B_{s}\left(\theta_{t}(\omega)\right)
$$

$B_{t}$ est une $M$-fonctionnelle au sens de [4], avec $M_{t}=1_{(S>t)}$. On rappelle que $S$ est terminal, parfait et effilé en tout point. On appelle $S^{(k)}$ les itérés de $S$ et l'on définit par récurrence

$$
\widetilde{B}_{S}(2)=B_{S}+B_{S}\left(\theta_{S}\right), \quad \widetilde{B}_{S^{(k)}}=\widetilde{B}_{S^{(k-1)}}+B_{S}\left(\theta_{\left.S^{(k-1)}\right)} ;\right.
$$

puis pour $t<\tilde{S}=\lim _{k} S^{(k)}$, il existe un $k$ tel que $S^{(k)} \leqq t<S^{(k+1)}$, et l'on pose

$$
\widetilde{B}_{t}=\widetilde{B}_{S^{(k)}}+B_{t-S^{(k)}}\left(\theta_{\left.S^{(k)}\right)} ;\right.
$$

on pose enfin $\widetilde{B}_{\tilde{S}}=\lim _{t \uparrow \tilde{S}} \widetilde{B}_{t}$. $\widetilde{B}_{t}$ est une fonctionnelle additive parfaite de $(X, \tilde{M})$ $\operatorname{avec} \tilde{M}_{t}=1_{(t<\tilde{S})}$.

Soient en effet des nombres $s, t$ tels que $t+s<\tilde{S}$; il existe des entiers $k$ et $l$ tels 
que $S^{(k)} \leqq t<S^{k+l}$ et $S^{(l)} \leqq t+s<S^{(l+1)}$ et d'après [4, p. 173], on a $S^{(l)}=t+S^{(l-k)} \circ \theta_{t}$. Commençons par montrer que

$$
\widetilde{B}_{S^{(l)}}=\widetilde{B}_{t}+\widetilde{B}_{S^{(l-k)}} \circ \theta_{t} \text { p.s.; }
$$

il suffit de montrer que

soit encore

$$
\widetilde{B}_{t-S^{(k)}}\left(\theta_{S^{(k)}}\right)+\widetilde{B}_{S^{(l-k)}}\left(\theta_{t}\right)=\widetilde{B}_{S^{(l-k)}}\left(\theta_{S^{(k)}}\right)
$$

$$
\widetilde{B}_{t-S^{(k)}}\left(\theta_{S^{(k)}}\right)+\widetilde{B}_{S^{(k+1)}}\left(\theta_{t}\right)=\widetilde{B}_{S^{(k+1)}}\left(\theta_{\left.S^{(k)}\right)}\right.
$$

D'après la propriété forte de Markov [4, p. 43], l'ensemble $\Lambda^{\prime}=\left\{\omega: \theta_{S(\omega)}(\omega) \in \Lambda\right\}$ est de probabilité nulle pour toute loi $P_{x}$. Soit $\omega$ fixé n'appartenant pas à $\Lambda^{\prime}$, il nous suffit de montrer la relation ci-dessus pour un tel $\omega$, soit encore pour tout $\omega \notin \Lambda$

$$
\widetilde{B}_{t-S^{(k)}(\omega)}(\omega)+\widetilde{B}_{S^{(k+1)}(\omega)}\left(\theta_{t-S^{k}(\omega)}(\omega)\right)=\widetilde{B}_{S^{(k+1)}(\omega)}(\omega),
$$

mais ceci est la relation d'additivité sur $(0, S)$. On a alors presque sûrement

$$
\begin{aligned}
\tilde{B}_{t+s} & =\widetilde{B}_{t}+\widetilde{B}_{S^{(l-k)}}\left(\theta_{t}\right)+\tilde{B}_{t+S-\left(t+S^{(l-k)_{0}} \theta_{t}\right.}\left(\theta_{S^{(l-k)}} \circ \theta_{t}\right) \\
& =\widetilde{B}_{t}+\widetilde{B}_{s}\left(\theta_{t}\right) .
\end{aligned}
$$

Maintenant il n'est pas difficile de voir que $\tilde{S}$ est un temps terminal parfait et effiilé en tout point et l'on peut recommencer avec $\tilde{S}$ ce qu'on vient de faire avec $S$ et continuer ainsi une récurrence transfinie, on appellera $\sigma^{(a)}$ les temps terminaux qui interviennent ainsi $\left(\sigma^{(1)}=S, \sigma^{(2)}=\widetilde{S}, \ldots\right)$ et $B^{(a)}$ les fonctionnelles correspondantes.

Soit $\eta$ une mesure de référence bornée, il existe un ordinal dénombrable $a$ tel que $P_{\eta}\left(\sigma^{(a)}=\zeta\right)=1$; en effet dans le cas contraire, comme $P_{\eta}\left(\sigma^{(a)}<\zeta\right)$ décroit il y aurait un ordinal $a$ à partir duquel $P_{\eta}\left(\sigma^{(a)}<\zeta\right)$ resterait constante et $>0$ ce qui est impossible car sur chaque trajectoire $\sigma^{(a)}$ devient égal à $\zeta$ pour un ordinal dénombrable. Comme $P .\left(\sigma^{(a)}<\zeta\right)$ est excessive, il en résulte qu'il existe un ordinal dénombrable $a$ tel que $\sigma^{(a)}=\zeta$, presque-sûrement. Soit alors $B^{(a)}$ la fonctionnelle correspondante; en plus de la relation d'additivité déjà montrée, il est clair qu'elle possède la propriété de continuité à droite, enfin $B_{t}^{(a)}$ est $\mathscr{F}_{t}$-mesurable; en effet $a$ étant dénombrable il suffit de montrer que $B_{t}^{(b)}$ est $\mathscr{F}_{t}$-mesurable pour $\sigma^{(b)} \leqq t<\sigma^{(b+1)}$ soit encore que $\widetilde{B}_{t}$ est $\mathscr{F}_{t}$-mesurable pour $S^{(k)} \leqq t<S^{k+1}$; cela se montre alors de la façon suivante: comme $\widetilde{B}_{S^{(k)}}$ est $\mathscr{F}_{t}$-mesurable il faut montrer que $B_{t-S^{(k)}}\left(\theta_{S^{(k)}}\right)$ est $\mathscr{F}_{t}$-mesurable; or $S^{(k)}$ est approchable par en dessous par des variables aléatoires $T^{n}$ ne prenant que des valeurs dyadiques $\left(T^{n}=i / 2^{n}\right.$ si $\left.i / 2^{n} \leqq S^{(k)}<(i+1) / 2^{n}\right)$ et la continuité à droite entraine que $B_{t-S^{(k)}}$ est limite de $B_{i-T^{n}}\left(\theta_{S^{k}}\right)$; de plus sur

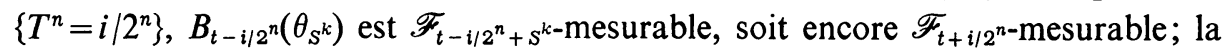
continuité à droite des $\mathscr{F}_{t}$ entraine la mesurabilité cherchée.

La fonctionnelle $B^{(a)}$ est donc une fonctionnelle additive du processus $X$ qui prolonge $B_{t}$.

DÉfInItIon. On dira que la fonctionnelle $B^{(a)}$ ainsi construite est la trace de $A$ sur $f$ et on la notera $A_{t}^{f}$. 
Si $A$ est dans $\Phi$, on a

$$
A_{t}^{f}=(f A)_{t}=\int_{0}^{t} f\left(X_{s}\right) d A_{s}
$$

Il est clair d'autre part que la trace de $A$ sur un ensemble $\Gamma$ ne charge que cet ensemble, et que la trace de la trace sur un sous-ensemble de $\Gamma$ est égale à la trace sur ce sous-ensemble.

II. La correspondance entre mesures et fonctionnelles additives.

II.1. Soit $A$ une fonctionnelle additive et $f \in b \mathscr{E}_{+}$; on pose

$$
\nu_{A}(f)=\sup _{t>0} \frac{1}{t} E_{\xi}\left((f A)_{t}\right)
$$

DÉfInItIon. Si $\nu_{A}(1)<\infty, A$ sera dite intégrable. Si $E$ est réunion d'une suite $\left\{E_{n}\right\}_{n \in N}$ d'ensembles de $\mathscr{E}$, tels que pour tout $n, \nu_{A}\left(1_{E_{n}}\right)<\infty, A$ sera dite $\sigma$-intégrable.

Proposition. (1) On a

$$
\nu_{A}(f)=\lim _{t \rightarrow 0} \frac{1}{t} E_{\xi}\left((f A)_{t}\right)=\lim _{\alpha \rightarrow \infty} \alpha \int \xi(d x) U_{A}^{\alpha} f(x),
$$

et la deuxième limite est une limite croissante.

(2) Si A est $\sigma$-intégrable, l'application $f \leadsto \nu_{A}(f)$ est une mesure positive et $\sigma$-finie que l'on désignera par $\nu_{A}$; elle est bornée si et seulement si A est intégrable. $\nu_{A}$ ne charge pas les ensembles polaires; si A est continue, $\nu_{A}$ ne charge pas les ensembles semi-polaires.

Démonstration. Soient $a<\nu_{A}(f)$ et $t_{0}$ tel que $E_{\xi}\left((f A)_{t_{0}}\right) / t_{0} \geqq a$; posons $t_{0}=n h+\delta$ avec $\delta<h ; \xi$ étant excessive on a en tenant compte de (I.3.1)

$$
\begin{aligned}
a \leqq \frac{1}{t_{0}} E_{\xi}\left((f A)_{t_{0}}\right) & \leqq \frac{1}{t_{0}} E_{\xi}\left((f A)_{n h}\right)+\frac{1}{t_{0}} E_{\xi}((f A)) \\
& \leqq \frac{n}{n h+\delta} E_{\xi}\left((f A)_{h}\right)+\frac{1}{t_{0}} E_{\xi}\left((f A)_{\delta}\right) .
\end{aligned}
$$

Supposons qu'il existe un $\delta>0$ tel que $E_{\xi}\left((f A)_{\delta}\right)=\infty$; comme pour tout $t$ il existe un entier $k$ tel que $k t \geqq \delta$, on a

$$
\infty=E_{\xi}\left((f A)_{\delta}\right) \leqq E_{\xi}\left((f A)_{k t}\right) \leqq k E_{\xi}\left((f A)_{t}\right),
$$

et par suite $\nu_{A}(f)=\lim _{t \rightarrow 0} E_{\xi}\left((f A)_{t}\right) / t=\infty$.

Si $E_{\xi}\left((f A)_{\delta}\right)<\infty$ pour tout $\delta>0$, la continuité à droite du processus $(f A)_{t}$ (rappelons que $f \in b \mathscr{E}_{+}$) entraine $\lim _{\delta \rightarrow 0} E_{\xi}\left((f A)_{\delta}\right)=0$. Passant à la limite dans (1) quand $h$ tend vers zéro, on obtient

$$
a \leqq \liminf _{k \rightarrow 0} \frac{1}{h} E_{\xi}\left((f A)_{h}\right) \leqq \limsup _{h \rightarrow 0} \frac{1}{h} E_{\xi}\left((f A)_{h}\right) \leqq \nu_{A}(f),
$$


ce qui démontre la première égalité; la seconde découle d'un théorème abélien [21]. La seconde limite est croissante, car pour $\beta \geqq \alpha$, on a d'après (I.3.2)

$$
\alpha U_{A}^{\alpha} \leqq \alpha U_{A}^{\beta}+\alpha(\beta-\alpha) U^{\alpha} U_{A}^{\beta},
$$

et comme $\xi$ est excessive

$$
\alpha\left\langle\xi, U_{A}^{\alpha}\right\rangle \leqq \alpha\left\langle\xi, U_{A}^{\beta}\right\rangle+(\beta-\alpha)\left\langle\xi, U_{A}^{\beta}\right\rangle=\beta\left\langle\xi, U_{A}^{\beta}\right\rangle .
$$

Supposons $A \sigma$-intégrable et soit $\left\{E_{n}\right\}_{n \in N}$, la suite d'ensemble tels que $\nu_{A}\left(1_{E_{n}}\right)<\infty$; l'application $f \rightarrow \nu_{A}\left(1_{E_{n}} f\right)$ est limite croissante des mesures $f \rightarrow \alpha\left\langle\xi, U_{A}^{\alpha}\left(1_{E_{n}} f\right)\right\rangle$; c'est donc une mesure bornée. De plus

$$
\nu_{A}(f)=\sup _{\alpha} \sup _{n} \alpha\left\langle\xi, U_{1_{E_{n} A}}^{\alpha}(f)\right\rangle=\sup _{n} \nu_{A}\left(1_{E_{n}} f\right)
$$

donc $\nu_{A}$ définit une mesure $\sigma$-finie, bornée si et seulement si $A$ est intégrable. La dernière phrase de l'énoncé est évidente.

Remarque 1 . On peut aussi montrer que $\nu_{A}(f)$ est limite croissante de la suite $2^{n} E_{\xi}\left((f A)_{2}-n\right)$.

Remarque 2. Si un ensemble porte $A$, il porte la mesure $\nu_{A}$ et inversement. Si $A$ est continue, $\nu_{A}$ ne charge aucun ensemble semi-polaire; le support de la mesure $\nu_{A}[4$, p. 204] est alors celui de la fonctionnelle $A$ [4, p. 212].

Remarque 3. Si $A$ est continue l'application $A \rightarrow \nu_{A}$ est injective comme le prouve le théorème de Motoo [4, p. 210]. Il n'en est pas de même lorsque $A$ est seulement naturelle comme le prouve le contre exemple de $[4$, p. 211].

REMARQue 4. La définition donnée dans [1] d'une fonctionnelle $\sigma$-intégrable est incorrecte car $1_{E} \cdot A$ n'est pas forcément une fonctionnelle additive. La définition de la mesure $\nu_{A}$ y est donc aussi incorrecte. Néanmoins les théorèmes limites restent valables puisqu'ils ne concernent que les fonctionnelles intégrables. La Proposition II.3 de [1] sera reprise et éclairée dans le présent article.

Remarquons encore qu'il n'y aurait pas de précautions à prendre si on se limitait aux fonctionnelles de $\Phi$.

II.2. Nous allons montrer que les mesures $\nu_{A}$ ainsi définies ont un certain nombre d'applications; il est donc important de connaitre la classe des fonctionnelles $\sigma$-intégrables et une bonne partie de cet article sera consacrée à leur caractérisation. Le lemme suivant sera essentiel à cet effet. Nous écrirons désormais FAI et FA $\sigma$ I pour fonctionnelle additive intégrable et $\sigma$-intégrable.

LeMmE. Si $B$ est une FAI, $A$ une fonctionnelle et $f \in b \mathscr{E}_{+}$et s'il existe un $\alpha$ tel que $U_{A}^{\alpha} f \leqq U_{B}^{\alpha}$, alors $\nu_{A}(f)<\infty$.

Démonstration. Pour $\beta \geqq \alpha$, l'équation résolvante et la relation (I.3.2) permettent d'écrire

$$
\beta\left\langle\xi, U_{B}^{\beta}-U_{A}^{\beta} f\right\rangle=\beta\left\langle\xi, U_{B}^{\alpha}-U_{A}^{\alpha} f\right\rangle-(\beta-\alpha)\left\langle\xi, \beta U^{\beta}\left(U_{B}^{\alpha}-U_{A}^{\alpha} f\right)\right\rangle ;
$$


comme $\xi$ est excessive, ceci implique

$$
\begin{aligned}
\beta\left\langle\xi, U_{B}^{\beta}-U_{A}^{\beta} f\right\rangle & \geqq \beta\left\langle\xi, U_{B}^{\alpha}-U_{A}^{\alpha} f\right\rangle-(\beta-\alpha)\left\langle\xi, U_{B}^{\alpha}-U_{A}^{\alpha} f\right\rangle \\
& =\alpha\left\langle\xi, U_{B}^{\alpha}-U_{A}^{\alpha} f\right\rangle,
\end{aligned}
$$

quelque soit $\beta \geqq \alpha$. On a donc $\beta\left\langle\xi, U_{B}^{\beta}\right\rangle \geqq \beta\left\langle\xi, U_{A}^{\beta} f\right\rangle$, ce qui démontre le lemme.

Remarque. Si $f \in \mathscr{L}_{+}^{1}(\xi) \cap b \mathscr{E}$, la fonctionnelle $A_{t}=\int_{0}^{t} f\left(X_{s}\right) d s$ est intégrable car on a toujours $E_{\xi}\left(A_{t}\right) / t \leqq \xi(f)$. Cet exemple et le lemme précédent nous permettront de montrer l'étendue de la classe des FA $\sigma \mathrm{I}$.

II.3. Proposition. Si $A$ est une FAI, $A \in \Phi$ ( $A_{t}$ est p.s. finie sur l'intervalle $[0, \zeta[)$.

Démonstration. Soit $R=\inf \left\{t: A_{t}=\infty\right\}$; si $P_{x}(R<\zeta)>0$

$$
U_{A}^{\alpha}(x)=E_{x} \int_{0}^{\zeta} e^{-\alpha t} d A_{t} \geqq E_{x}\left(e^{-\alpha R} A^{R}\right)=\infty ;
$$

or $U_{A}^{\alpha}(x)$ est fini $\xi$-presque partout puisque $\nu_{A}(E)=\lim _{\alpha \rightarrow \infty} \alpha\left\langle\xi, U_{A}^{\alpha}\right\rangle<\infty$, on a donc $P_{x}(R<\zeta)=0 \xi$-p.s. Mais $R$ est un temps d'arrêt effilé en tout point et terminal fort [4, p. 127]; la fonction $P .(R<\zeta)$ est donc excessive et comme $\xi$ est une mesure de référence $P_{x}(R<\zeta)=0$ pour tout $x$ de $E$.

III. Fonctionnelles continues.

III.1. Nous allons d'abord étudier les fonctionnelles continues pour lesquelles on peut donner des résultats assez complets sans hypothèse de dualité.

THÉORÈme. Toute fonctionnelle continue A est $\sigma$-intégrable. De plus il existe une suite croissante $\left\{E_{n}\right\}$ d'ensembles presque-boréliens de réunion $E$ tels que les potentiels $U_{A}^{\alpha}\left(x, E_{n}\right)$ soient intégrables et bornés. La trace de $A$ sur $E_{n}$ est de potentiel intégrable et borné, et la mesure associée est la trace sur $E_{n}$ de la mesure associée à A. Enfin si l'on pose $T_{n}=T_{E_{n}^{c}}$ et $T=\lim _{n} T_{n}$, T est presque sûrement positif et

$$
T=R=\inf \left\{t: A_{t}=\infty\right\} .
$$

Démonstration. Soit $f$ une fonction de $\mathscr{L}^{1}(\xi) \cap b \mathscr{E}_{+}$partout positive, et posons

on a $0<\phi \leqq\|f\|$ et

$$
\phi(x)=E_{x} \int_{0}^{\zeta} e^{-t} f\left(X_{t}\right) \exp \left[-A_{t}\right] d t ;
$$

$$
\begin{aligned}
U_{A}^{1} \phi(x) & =E_{x} \int_{0}^{\zeta} e^{-t} E_{X_{t}} \int_{0}^{\zeta} e^{-s} f\left(X_{s}\right) \exp \left[-A_{s}\right] d s d A_{t} \\
& =E_{x} \int_{0}^{\zeta} e^{-t} d A_{t} \int_{0}^{\zeta} e^{-s} f\left(X_{s+t}\right) \exp \left[-\left(A_{s+t}-A_{t}\right)\right] d t \\
& =E_{x} \int_{0}^{\zeta} e^{-s} f\left(X_{s}\right) \exp \left[-A_{s}\right] d s \int_{0}^{s} \exp \left[A_{t}\right] d A_{t} \\
& =E_{x} \int_{0}^{\zeta} e^{-s} f\left(X_{s}\right) \exp \left[-A_{s}\right]\left(\exp \left[A_{s}\right]-1\right) d s \\
& =E_{x} \int_{0}^{\zeta} e^{-s} f\left(X_{s}\right)\left(1-\exp \left[-A_{s}\right]\right) d s \leqq U^{1} f(x) .
\end{aligned}
$$


Soit maintenant $E_{n}=\{x: \phi(x) \geqq 1 / n\} ; E_{n}$ est un fermé fin presque-borélien, $E=\bigcup_{n} E_{n}$ et $U_{A}^{1}\left(x, E_{n}\right)$ est intégrable et borné car

$$
E_{x} \int_{0}^{\zeta} e^{-t} 1_{E_{n}}\left(X_{t}\right) d A_{t} \leqq E_{x} \int_{0}^{\zeta} e^{-t} n \phi\left(X_{t}\right) d A_{t} \leqq n U^{1} f(x)
$$

on conclut alors en utilisant le Lemme II.2. Soit maintenant $A^{n}=A^{E_{n}}$ la trace de $A$ sur $E_{n}$; on peut définir $\phi^{n}(x)$ pour $A^{n}$ comme on a défini $\phi(x)$ pour $A$ et le même calcul donne $U_{A^{n}}^{1} \phi^{n}(x) \leqq U^{1} f(x)$. Il est clair que $\phi^{n} \geqq \phi$, donc que $1_{E_{n}} \leqq n \phi^{n}$ et le même calcul conduit à

$$
U_{A^{n}}^{1}(x)=U_{A}^{1}\left(x, E_{n}\right) \leqq n U^{1} f(x) ;
$$

la fonctionnelle $A^{n}$ est donc intégrable et de potentiel borné, D'autre part $A_{t}^{n}$ et $\left(1_{E_{n}} \cdot A\right)_{t}$ coïncident pour $t<R$, et par suite la différence des expressions $\alpha\left\langle\xi, U_{A}^{\alpha} 1_{\Gamma}\right\rangle$ et $\alpha\left\langle\xi, U_{A}^{\alpha}\left(x, E_{n}\right) \cap \Gamma\right\rangle$ où $\Gamma \in \mathscr{E}$, est égale à

$$
\left\langle\xi, E_{x} \int_{R}^{\zeta} \alpha e^{-\alpha t} 1_{\Gamma}\left(X_{t}\right) d A_{t}^{n}\right\rangle \text {. }
$$

Un calcul comme le précédent montre que

$$
\begin{aligned}
E_{x} \int_{R}^{\zeta} \alpha e^{-\alpha t} 1_{\Gamma}\left(X_{t}\right) d A_{t}^{n} & \leqq n E_{x} \int_{R}^{\zeta} \alpha e^{-\alpha t} f\left(X_{t}\right) d t \\
& \leqq n\|f\| E_{x}\left(e^{-\alpha R}\right)
\end{aligned}
$$

comme $R$ est $P_{x}$ p.s. strictement positif, cette expression décroit vers zéro lorsque $\alpha$ tend vers l'infini; maintenant quelque soit $\alpha$

$$
\begin{aligned}
n\left\langle\xi, E_{x} \int_{R}^{\zeta} \alpha e^{-\alpha t} f\left(X_{t}\right) d t\right\rangle & \leqq n\left\langle\xi, E_{x} \int_{R}^{\zeta} \alpha e^{-\alpha t} f\left(X_{t}\right) d t\right\rangle \\
& \leqq n\langle\xi, f\rangle,
\end{aligned}
$$

cette expression tend donc vers zéro et par suite (1) tend vers zéro lorsque $\alpha$ tend vers l'infini. La mesure $\nu_{A^{n}}$ est donc la trace sur $E_{n}$ de la mesure $\nu_{A}$.

Dans ce qui précède nous aurions pu prendre $E_{n}=\{x: \phi(x)>1 / n\}$, c'est à dire $E_{n}$ ouvert fin, et arriver aux mêmes conclusions; il est donc bien clair que, dans les deux cas, pour tout $x$ de $E$ il existe un $n$ tel que $P_{x}\left(T_{n}>0\right)=1, T$ est donc presquesûrement strictement positif. Dans les deux cas on a aussi $\phi\left(X_{T_{n}}\right) \leqq 1 / n$ presque sûrement sur $\left(T_{n}<\zeta\right)$, soit

$$
1_{\left(T_{n}<\zeta\right)} E_{X_{T_{n}}} \int_{0}^{\zeta} e^{-t} f\left(X_{t}\right) e^{-A_{t}} d t \leqq \frac{1}{n},
$$

ou encore $P_{x}$ p.s. pour tout $x$ de $E$

$$
1_{\left(T_{n}<\zeta\right)} \exp \left[T_{n}\right] \exp \left[-A_{T_{n}}\right] E_{x}\left(\int_{T_{n}}^{\zeta} e^{-t} f\left(X_{t}\right) \exp \left[-A_{t}\right] d t \mid \mathscr{F}_{T_{n}}\right) \leqq \frac{1}{n}
$$


Soit $\bigvee_{n} \mathscr{F}_{T_{n}}$ la tribu engendrée par la réunion des $\mathscr{F}_{T_{n}}$, lorsque $T_{n}$ tend vers $T$, l'intégrale $\int_{T_{n}}^{\zeta} e^{-t} f\left(X_{t}\right) \exp \left[-A_{t}\right] d t$ tend vers $\int_{T}^{\infty} e^{-t} f\left(X_{t}\right) \exp \left[-A_{t}\right] d t$ en restant bornée par $\|f\|$, on peut donc passer à la limite (cf. [13]) pour obtenir

$$
1_{(T \leqq \zeta)} e^{T} \exp \left[-A_{T}\right] E_{x}\left(\int_{T}^{\zeta} e^{-t} f\left(X_{t}\right) \exp \left[-A_{t}\right] d t \mid \bigvee_{n} \mathscr{F}_{T_{n}}\right)=0
$$

$P_{x}$-presque sûrement pour tout $x$ de $E$. Sur $\{T<R\}, e^{T} \exp \left[-A_{T}\right]$ est strictement positif, et comme $f$ est strictement positive l'espérance conditionnelle est strictement positive et on arrive à une contradiction. On a donc $T \geqq R$; mais d'autre part les expressions $\left(1_{E_{n}} \cdot A\right)_{t}$ sont majorées par $A_{t}^{n}$ donc finies sur [0, $\zeta[$ d'après II.3, par suite $A_{T_{n} \wedge t}=\left(1_{E_{n}} \cdot A\right)_{T_{n} \wedge t}$ est fini; $A_{t}$ est donc fini sur l'intervalle [0,T[ et $T=R$ presque sûrement.

III.2. Corollaire. A appartient à $\Phi_{c}$ si et seulement si $T \geqq \zeta$ presque sûrement.

Sous l'hypothèse $(\mathrm{F})$ de Hunt, Blumenthal et Getoor [5] ont étudié une classe de FAC dites "smooth" qui est contenue dans la classe $\Phi_{c}$. Cette classe coïncide avec la classe $\Phi_{c}$ si $X$ est récurrent comme cela résulte de [2].

III.3. Dans le suite nous appellerons $\tau_{t}$ le changement de temps associé à la fonctionnelle $A_{t}, \tilde{X}_{t}$ le processus de Markov $X_{\tau_{t}}$ dont le semi-groupe est $P_{\tau_{t}}$ et la résolvante $V_{A}^{\alpha} f(x)=E_{x} \int_{0}^{\infty} \exp \left[-\alpha A_{t}\right] f\left(X_{t}\right) d A_{t}$.

Proposition. La mesure $\nu_{A}$ est une measure excessive de référence pour le processus $\tilde{X}$.

Démonstration. Si $\Gamma \in \mathscr{E}$ et $\nu_{A}(\Gamma)=0$, par définition de $\nu_{A}$, pour tout $\alpha>0$ on a $\left\langle\xi, U_{A}^{\alpha}(\Gamma)\right\rangle=0$, soit $U_{A}^{\alpha} \Gamma(x)=E_{x} \int_{0}^{R} e^{-\alpha t} f\left(X_{t}\right) d A_{t}=0$ puisque $\xi$ est une mesure de référence pour $X$. Comme exp $\left[-\alpha A_{t}\right]$ est positif sur [0, $R$ [ et que $A_{t}$ ne charge pas $[R, \infty[$, ceci est équivalent à

$$
V_{A}^{\alpha} \Gamma(x)=E_{x} \int_{0}^{R} \exp \left[-\alpha A_{t}\right] f\left(X_{t}\right) d A_{t}=0
$$

pour tout $x$ de $E . \nu_{A}$ est donc bien une mesure de référence pour $\tilde{X}$; pour montrer qu'elle est excessive il suffit de montrer que $\left\langle\nu_{A}, V_{A}^{1} f\right\rangle \leqq\left\langle\nu_{A}, f\right\rangle$ où $f$ est une fonction borélienne positive. Or

$$
\begin{aligned}
\left\langle\nu_{A}, V_{A}^{1} f\right\rangle & =\lim _{s \downarrow 0} \frac{1}{s} E_{\xi}\left(\int_{0}^{s}\left(E_{X_{u}} \int_{0}^{\infty} \exp \left[-A_{t}\right] f\left(X_{t}\right) d A_{t}\right) d A_{u}\right) \\
& =\lim _{s \downarrow 0} \frac{1}{s} E_{\xi}\left(\int_{0}^{s} d A_{u} \int_{0}^{\infty} \exp \left[-A_{t} \circ \theta_{u}\right] f\left(X_{t+u}\right) d A_{t+u}\right) \\
& =\lim _{s \downarrow 0} \frac{1}{s} E_{\xi}\left(\int_{0}^{s} \exp \left[A_{u}\right] d A_{u} \int_{u}^{\infty} \exp \left[-A_{t}\right] f\left(X_{t}\right) d A_{t}\right) \\
& =\lim _{s \downarrow 0} \frac{1}{S} E_{\xi}\left(\int_{0}^{s} \exp \left[-A_{t}\right] f\left(X_{t}\right) d A_{t} \int_{0}^{t} \exp \left[A_{u}\right] d A_{u}\right. \\
& \left.\quad+\int_{s}^{\infty} \exp \left[-A_{t}\right] f\left(X_{t}\right) d A_{t} \int_{0}^{s} \exp \left[A_{u}\right] d A_{u}\right)
\end{aligned}
$$




$$
\begin{aligned}
=\lim _{s \downarrow 0} \frac{1}{s} E_{\xi}\left(\int_{0}^{s} \exp \left[-A_{t}\right] f\left(X_{t}\right)\left(\exp \left[A_{t}\right]-1\right) d A_{t}\right. \\
\left.+\left(\exp \left[A_{s}\right]-1\right) \int_{s}^{\infty} \exp \left[-A_{t}\right] f\left(X_{t}\right) d A_{t}\right) \\
=\lim _{s \downarrow 0} \frac{1}{s} E_{\xi}\left(\int_{0}^{s} f\left(X_{t}\right) d A_{t}-\int_{0}^{\infty} \exp \left[-A_{t}\right] f\left(X_{t}\right) d A_{t}\right. \\
\left.+\exp \left[A_{s}\right] \int_{s}^{\infty} \exp \left[-A_{t}\right] f\left(X_{t}\right) d A_{t}\right) ;
\end{aligned}
$$

d'autre part

$$
\begin{aligned}
E_{\xi}\left(e^{A_{s}} \int_{s}^{\infty} \exp \left[-A_{t}\right] f\left(X_{t}\right) d A_{t}\right) & =E_{\zeta P_{s}}\left(\int_{0}^{\infty} \exp \left[-A_{t}\right] f\left(X_{t}\right) d A_{t}\right) \\
& \leqq E_{\xi}\left(\int_{0}^{\infty} \exp \left[-A_{t}\right] f\left(X_{t}\right) d A_{t}\right)
\end{aligned}
$$

puisque $\xi$ est excessive; en rapprochant ceci de la formule précédente on trouve

$$
\left\langle\nu_{A}, V_{A}^{1} f\right\rangle \leqq \lim _{s \downarrow 0} \frac{1}{s} E_{\xi}\left(\int_{0}^{s} f\left(X_{t}\right) d A_{t}\right)=\left\langle\nu_{A}, f\right\rangle .
$$

III.4. Dans le cas récurrent où $\xi$ est la mesure invariante du processus (cf. [1] et [2]) et si $A \in \Phi_{c}$ les inégalités sont des égalités et $\nu_{A}$ est invariante. La proposition suivante, montre à quelle condition le processus balayé $\tilde{X}$ est récurrent.

Proposition. Si X est finement récurrent et si A est une FACoI qui n'appartient pas à $\Phi_{c}$, le processus $\tilde{X}$ correspondant est transient. Si A est dans $\Phi_{c}$, le processus $\tilde{X}$ est récurrent au sens de Harris par rapport à la mesure $\nu_{A}$ et dans ce cas $\nu_{A}$ est la seule mesure excessive pour $P_{\tau_{t}}$.

Démonstration. Dans le cas récurrent il résulte de [3] que $P_{x}(R<\infty)=0$ ou 1 . Si $A$ n'est pas dans $\Phi, P_{x}(R<\infty)=1$ et $\tau_{t}$ croit vers $R$ presque-sûrement. La propriété de Blumenthal assure alors que $X_{\tau_{t}}$ tend vers $X_{R}$ presque-sûrement. Soit maintenant $y_{1}$ et $y_{2}$ deux points de $E$, et $v_{1}$ et $v_{2}$ deux voisinages fermés disjoints de $y_{1}$ et $y_{2}$; pour tout $x$ de $E$ on a:

$$
P_{x}\left(\limsup _{t \rightarrow \infty} 1_{v_{i}}\left(X_{\tau_{t}}\right)=1\right)=P_{x}\left(1_{v_{i}}\left(X_{T}\right)=1\right)
$$

comme $P_{x}\left(1_{v_{1} \cap v_{2}}\left(X_{R}\right)=1\right)=0$, le processus partant de $x$ ne peut récurrer dans $v_{1}$ et $v_{2}$.

Inversement si $A$ est dans $\Phi_{c}$, et si $\Gamma$ est un ensemble le processus

$$
B_{t}=\int_{0}^{t} 1_{\Gamma}\left(X_{s}\right) d A_{s}
$$


est continu et fini et défini donc une FAC de $X$. Comme $\nu_{A}(\Gamma)=E_{\xi}\left(B_{t}\right) / t$, si $\nu_{A}(\Gamma)>0, B_{\infty}$ ne peut être nulle $P_{\xi}$-p.s., il résulte alors de [1] que $B_{\infty}=\infty$ p.s.; or

$$
\int_{0}^{\infty} 1_{\Gamma}\left(X_{\tau_{t}}\right) d t=\int_{0}^{\infty} 1_{\Gamma}\left(X_{s}\right) d A_{s}
$$

et par suite pour tout $x$ de $E, P_{x}\left(\int_{0}^{\infty} 1_{\Gamma}\left(X_{\tau_{t}}\right) d t=\infty\right)=1$.

Remarque. Si $A$ est dans $\Phi$ et si son support est fermé dans $E, \tilde{X}$ est un processus standard et il résulte alors de [2] et de la Proposition III.3 qu'il est finement récurrent. Ceci est en particulier vérifié lorsque $A$ est strictement croissante; il n'est pas difficile de montrer par des considérations de support que ceci a lieu si et seulement si $\nu_{A}$ charge tous les ouverts fins.

IV. Fonctionnelles discontinues. Pour les fonctionnelles discontinues, même naturelles, on ne peut pas donner de résultat comparable au Théorème III.1. Dans ce paragraphe nous allons donner quelques premiers résultats tendant à caractériser les $\mathrm{FAN} \sigma \mathrm{I}$, ce qui sera fait complètement sous les hypothèses de dualité.

IV.1. Proposition. Pour que A soit une FAfI, il est nécessaire que les sauts de A soient presque sûrement finis.

Démonstration. Soit $S=\inf \left\{t: A_{t}-A_{t^{-}}=\infty\right\}$; le fonction $P .(S \leqq \zeta)$ est excessive et par suite dire que $A$ est p.s. à sauts finis c'est à dire que $P_{\xi}(S<\zeta)=0$. Si $A$ est $\sigma$-intégrable, il existe une suite $E_{n}$ d'ensembles croissant vers $E$ et tels que $\nu_{A}\left(E_{n}\right)<\infty$ ce qui entraine

$$
E_{\xi} \int_{0}^{\zeta} e^{-t} 1_{E_{n}}\left(X_{t}\right) d A_{t}<\infty
$$

puis

$$
P_{\xi}\left(\left\{1_{E_{n}}\left(X_{S}\right)>0\right\} \cap\{S<\zeta\}\right)=0 .
$$

En faisant tendre $n$ vers l'infini, on obtient $P_{\xi}(S<\zeta)=0$.

Remarque. Sous l'hypothèse $(F)$ de Hunt nous montrerons que cette condition est également suffisante. L'exemple suivant montre que ce résultat ne peut s'étendre.

L'espace des états est un sous-ensemble du plan euclidien constitué par le demi-axe $E_{0}$ des $x$ positifs ou nuls et par les demi-droites $E_{n}=\{(x, y), x<0, y=n x\}$. Le processus est celui de la translation uniforme sur toutes ces droites. Soit $p_{n}$ le terme général d'une série de somme 1 , la mesure égale à $p_{n} \lambda$ sur $E_{n}$, et à $\lambda$ sur $E_{0}$ ( $\lambda$ étant la mesure de Lebesgue sur les demi-droites) est invariante et servira de mesure de référence. Soit alors $A_{t}$ la fonctionnelle additive qui a un saut unique de hauteur $1 / p_{n}$ lorsque le processus passe en $(0,0)$ en venant de $E_{n}$; il est facile de voir que la mesure $v_{A}$ donne au point $(0,0)$ une masse infinie.

Cet exemple montre aussi que la même mesure peut correspondre à plusieurs FAN lorsqu'elles ne sont pas continues.

IV.2. Nous allons toutefois donner des exemples de FAN $\sigma$ I discontinues qui serviront dans la suite. 
Proposition. Si $\tau$ est un temps d'arrêt terminal, accessible et effilé en tout point, $B^{\tau}$ est une FANoI. Il existe de plus une suite croissante $\left\{E_{n}\right\}$ de fermés fins presque boréliens de réunion $E$, tels que les potentiels $U_{B^{r}}^{1}\left(x, E_{n}\right)$ soient intégrables et bornés.

Démonstration. Soient $\left\{B_{n}\right\}$ une suite d'ensembles boréliens de réunion $E$ et de $\xi$-mesure finie; on pose $f_{n}(x)=U^{1}\left(x, B_{n}\right)$. Si $\Phi(x)=E_{x}\left(e^{-\imath}\right)$ on a $\Phi<1$, et par suite si $\left\{u_{n}\right\}$ est une suite de nombres réels croissant vers 1 en restant strictement inférieurs, les ensembles $K_{n}=\left\{x: \Phi(x) \leqq u_{n} f_{n}(x)\right\}$ sont des fermés fins presque boréliens de réunion $E$. Soit $f$ une fonction borélienne bornée $\xi$-intégrable et strictement positive $\left(f=\sum_{n>0} 1_{B_{n}} / 2^{n} \xi\left(B_{n}\right)\right.$ par exemple); les ensembles

$$
H_{n}=\left\{x: U^{1} f(x) \geqq 1 / n\right\}
$$

sont des fermés fins presque boréliens de réunion $E$ et par suite les ensembles $E_{n}=H_{n} \cap K_{n}$ sont aussi des fermés fins presque boréliens de réunion $E$. Les $E_{n}$ répondent à la question; en effet soit $\tau^{\prime}$ le temps d'arrêt égal à

$$
\inf \left\{t: B_{t}^{\tau}-\neq B_{t}^{\tau} \text { et } X_{t} \in E_{n}\right\}
$$

si cet ensemble n'est pas vide et à $+\infty$ sinon, on a $\tau^{\prime} \geqq \tau$ et

$$
U_{B^{\tau}}^{1}\left(x, E_{n}\right)=P_{\tau^{\prime}}^{1} 1(x)+P_{\tau^{\prime}}^{1} P_{\tau^{\prime}}^{1} 1(x)+\cdots+P_{\tau^{\prime}}^{1} P_{\tau^{\prime}}^{1} \cdots P_{\tau^{\prime}}^{1} 1(x)+\cdots ;
$$

or $P_{\tau^{\prime}}^{1} 1 \leqq P_{\tau}^{1} 1 \leqq u_{n} f_{n}$ sur $E_{n}$; comme les mesures $P_{\tau^{\prime}}^{1}(x, \cdot)$ sont concentrées sur le fermé fin $E_{n}$

$$
P_{\tau^{\prime}}^{1} P_{\tau^{\prime}}^{1} 1 \leqq P_{\tau^{\prime}}^{1} 1\left(\sup _{x} P_{\tau^{\prime}}^{1} 1\right) \leqq u_{n}^{2} f_{n} \quad \text { sur } E_{n},
$$

et par récurrence $\left(P_{\tau^{\prime}}^{1}\right)^{k} 1 \leqq u_{n}^{k} f_{n}$ sur $E_{n}$. D'autre part $\tau^{\prime}$ étant un temps terminal effilé en tout point les fonctions $\left(P_{\tau^{\prime}}^{1}\right)^{k} 1$ sont 1 -excessives et comme $\tau^{\prime} \geqq T_{E_{n}}$, le théorème du balayage donne

$$
P_{\tau^{\prime}}^{1}\left(P_{\tau^{\prime}}^{1}\right)^{k} 1 \leqq P_{T_{E_{n}}}^{1}\left(P_{\tau^{\prime}}^{1}\right)^{k} 1 \leqq u_{n}^{k} f_{n} \quad \text { partout, }
$$

(on rappelle que $f_{n}$ a été choisie 1-excessive); on a donc

$$
U_{A}^{1}\left(x, E_{n}\right)-P_{\tau^{\prime}}^{1} 1 \leqq f_{n} /\left(1-u_{n}\right) .
$$

D'autre part $X_{\tau^{\prime}} \in E_{n}$ sur $\left\{\tau^{\prime}<\infty\right\}$, par suite

$$
\begin{aligned}
P_{\tau^{\prime}}^{1} 1(x) & \leqq E_{x}\left(e^{-\tau^{\prime}} 1_{E_{n}}\left(X_{\tau^{\prime}}\right)\right) \leqq E_{x}\left(e^{-\tau^{\prime} 1_{H_{n}}}\left(X_{\tau^{\prime}}\right)\right) \\
& \leqq n E_{x}\left(e^{-\tau^{\prime}} U^{1} f\left(X_{\tau^{\prime}}\right)\right) \leqq n U^{1} f .
\end{aligned}
$$

On a donc finalement

$$
U_{B^{r}}^{1}\left(x, E_{n}\right) \leqq n U^{1} f(x)+U^{1}\left(x, B_{n}\right) /\left(1-u_{n}\right),
$$

et on conclut en appliquant le Lemme II.2.

Remarque 1. Soit $H$ un ensemble effilé, non polaire. $T_{H}$ est un temps terminal effilé en tout point; la fonctionnelle $B^{T_{H}}$ n'est pas forcément naturelle car $T_{H}$ n'est pas forcément accessible si l'on ne fait pas l'hypothèse (B) de Hunt. Le raisonnement précédent reste néanmoins valable, et $B^{T_{H}}$ est une FAoI; la mesure corre- 
spondante est concentrée sur $H$, car si $g$ est borélienne et si $T^{(n)}$ désigne les itérés de $T_{H}$

$$
g B_{t}^{T_{H}}=\sum_{n>0} g\left(X_{T}(n)\right) 1_{\left(T^{(n)} \leqq t ; T^{(n)}<\zeta\right)} .
$$

IV.3( $\left.{ }^{2}\right)$. On sait $(\S \mathrm{I})$ que $\int_{0}^{t} 1_{E_{n}}\left(X_{s}\right) d B_{s}^{\tau}$ n'est pas forcément une fonctionnelle additive et l'on ne peut définir la trace de $B^{\tau}$ sur $E_{n}$ comme cela a été fait au $\S I$ car le raisonnement de [7] ne prouve pas que $B$ soit parfaite si $B^{\imath}$ n'est pas dans $\Phi$ (toujours par ce que $\left(1_{E_{n}} B^{\imath}\right)$ n'est pas une FAN). Nous allons donc ici définir directement cette trace, ce qui prouvera en outre que le raisonnement de [7] s'étend aux fonctionnelles additives à sauts finis.

Pour tout ordinal dénombrable, on définit les itérés de $\tau$ par

$$
\tau^{(a)}=\tau^{(a-1)}+\tau \circ \theta_{\tau^{(a-1)}}
$$

si $a$ est de première espèce, et par

$$
\tau^{(b)}=\lim _{a<b} \tau^{(a)}
$$

si $b$ est de 2ème espèce; nous noterons $\mathscr{A}$ l'ensemble des ordinaux de lère espèce. Nous poserons $\nu_{n}=\inf \left\{\tau^{(a)}: a \in \mathscr{A}, X_{\tau}(a) \in E_{n}\right\}$ si cet ensemble n'est pas vide, $\nu_{n}=+\infty$ sinon; $\nu_{n}$ est un temps d'arrêt effilé en tout point et de plus il est terminal, car si $\tau^{(a)}>t$, il existe par les raisonnements de [4, p. 173] et [12, partie IV], un ordinal $\alpha$ de $\mathscr{A}$ tel que $\tau^{(a)}=t+\tau^{(\alpha)} \circ \theta_{t}$; on peut donc écrire si $\nu_{n}>t$

$$
\begin{aligned}
& \nu_{n}=\inf \left\{t+\tau^{(\alpha)} \circ \theta_{t}: X_{t+\tau^{(\alpha)}{ }_{0} \theta_{t}} \in E_{n}\right\} \\
& =t+\inf \left\{\tau^{(\alpha)}: X_{\tau}^{(\alpha)} \circ \theta_{t} \in E_{n}\right\}=t+\nu_{n} \circ \theta_{t} \text {. }
\end{aligned}
$$

On peut donc associer à $\nu_{n}$ la fonctionnelle $B^{v_{n}}$. Le 1-potentiel de cette fonctionnelle est supérieur à $U_{B^{\tau}}^{1}\left(x, E_{n}\right)$, mais dans la démonstration de IV.2 nous nous sommes servis uniquement de $\tau^{\prime} \geqq \tau$; or on a encore $\nu_{n} \geqq \tau$, le même raisonnement prouve donc que le 1-potentiel de $B^{v_{n}}$ est intégrable et borné et que $B^{v_{n}}$ est intégrable et par suite de la classe $\Phi$.

La fonctionnelle $B^{v_{n}}$ peut aussi se décrire autrement. La suite des temps d'arrêt $\tau^{(a)}$ est croissante et par un raisonnement fait en I.7 il existe un ordinal dénombrable $\alpha$ tel que $\tau^{(\alpha)}=\zeta$ presque sûrement. On appelle alors $d B_{t}$ la mesure aléatoire

et l'on a

$$
d B_{t}(\omega)=\sum_{a \in \mathscr{A} ; a<\alpha} \varepsilon_{\tau}^{(a)}(d t)
$$

$$
B_{t^{n}}^{v^{n}}=\int_{0}^{t} 1_{E_{n}}\left(X_{s}\right) d B_{s}
$$

Le raisonnement fait au paragraphe III pour les fonctionnelles continues prouve que la mesure associée à $B^{v_{n}}$ est la trace sur $E_{n}$ de la mesure associée à $B^{\tau}$. D'autre

${ }^{(2)}$ Ce paragraphe m'a été suggéré par P. A. Meyer. 
part $B^{\tau}$ est limite croissante des fonctionnelles finies $B^{v_{n}}$, elle est donc équivalente à une fonctionnelle parfaite.

IV.4. L'exemple du paragraphe IV.1 prouve aussi qu'une FAN discontinue de potentiel fini peut ne pas être $\sigma$-intégrable; on a toutefois le résultat suivant en supposant $X$ standard spécial (Hypothèse IV.4.1 de [4]).

Proposition. Les FAN de potentiel borné sont $\sigma$-intégrables.

Démonstration. Soit $A$ une FAN telle que $U_{A}^{1}(x) \leqq M<\infty$ pour tout $x$ de $E$ et soit $f$ une fonction borélienne bornée $\xi$-intégrable et strictement positive; on pose $K_{n}=\left\{x: U_{A}^{1}(x) \leqq n U^{1} f(x)\right\}$ et $H_{n}=\left\{x: U^{1} f(x) \geqq 1 / n\right\}$ puis $F_{n}=H_{n} \cap K_{n}$. On a $\bigcup_{n} F_{n}=E$, et

$$
\begin{aligned}
U_{A}^{1}\left(x, F_{n}\right) & =E_{x}\left(\int_{\left(T_{F_{n}}, \infty\right.} e^{-t} 1_{F_{n}}\left(X_{t}\right) d A_{t}\right) \\
& \leqq E_{x}\left(\exp \left[-T_{F_{n}}\right]\left(A_{T_{F_{n}}}-A_{T_{F_{n}}^{-}}\right)\right)+P_{F_{n}}^{1} U_{A}^{1}\left(x, F_{n}\right)
\end{aligned}
$$

mais d'après [4, p. 180] ou [9, p. 184], $\left(A_{T_{F_{n}}} A_{T_{F_{n}}^{-}}\right) \leqq M$ et par suite

$$
\begin{aligned}
U_{A}^{1}\left(x, F_{n}\right) & \leqq M E_{x}\left(\exp \left[-T_{F_{n}}\right]\right)+n P_{F_{n}}^{1} U^{1} f(x) \\
& \leqq M E_{x}\left(\exp \left[-T_{F_{n}}\right] 1_{F_{n}}\left(X_{T_{F_{n}}}\right)\right)+n P_{F_{n}}^{1} U^{1} f(x) \\
& \leqq n M E_{x}\left(\exp \left[-T_{F_{n}}\right] U^{1} f\left(X_{T_{F_{n}}}\right)\right)+n P_{F_{n}}^{1} U^{1} f(x) \\
& \leqq n(M+1) P_{F_{n}}^{1} U^{1} f(x) \leqq n(M+1) U^{1} f(x) ;
\end{aligned}
$$

on conclut alors en utilisant le Lemme II.2.

IV.5. Nous appellerons $\mathscr{M}$ le cone des mesures associées aus FAI naturelles ou non. On sait que les mesures de $\mathscr{M}$ ne charge pas les ensembles polaires.

Proposition. Un ensemble est polaire si et seulement si il est de mesure nulle pour toutes les mesures de $\mathscr{M}$.

Démonstration. Seule la suffisance est à démontrer. Si un ensemble n'est pas polaire il contient un compact $K$ non polaire. Montrons que $K$ est chargé par une mesure de $\mathscr{M}$.

(a) Si $K-K_{r}$ est polaire, $K$ est projectif [4, p. 230] et par suite la balayée sur $K$ d'une FACI strictement croissante est une FAC intégrable d'après le Lemme II.2., non nulle, et portée par $K_{r} \subset K$.

(b) Si $K-K_{r}$ n'est pas polaire, il contient un ensemble effilé non-polaire, qui porte une mesure de $\mathscr{M}$ d'après la remarque qui termine le paragraphe IV.2. et le paragraphe IV.3.

Remarque 1. Sous l'hypothèse (B) de Hunt on peut restreindre le cone $\mathscr{M}$ au cone des mesures associées aux FANI et obtenir le même résultat.

REMARQUE 2. Le même théorème pour les semi-polaires a été démontré par Dellacherie. 


\section{Les hypotheses de dualité.}

V.0. Dans toute la suite de cet article nous supposerons donnés deux processus $X$ et $\hat{X}$ en dualité, c'est à dire qu'il existe une mesure $\xi \sigma$-finie, et des fonctions $U^{\alpha}(x, y), \alpha \geqq 0$, telles que

(i) $U^{\alpha}(x, \Gamma)=\int_{\Gamma} U^{\alpha}(x, y) \xi(d y), \hat{U}^{\alpha}(\Gamma, y)=\int_{\Gamma} \xi(d x) U^{\alpha}(x, y)$;

(ii) les fonctions $x \rightarrow U^{\alpha}(x, y)$ sont $\alpha$-excessives pour $X$;

(iii) les fonctions $y \rightarrow U^{\alpha}(x, y)$ sont $\alpha$-excessives pour $\hat{X}$, c'est à dire co-excessives. (De façon générale les objets relatifs à $\hat{X}$ seront précédés du préfixe co- ou surmontés d'un chapeau.)

Nous renvoyons à [4] pour tout ce qui concerne ces hypothèses. Remarquons que nous ne faisons aucune hypothèse sur les résolvantes, en particulier nous ne les supposons pas fortement felleriennes.

La mesure $\xi$ est une mesure excessive de référence, et peut donc servir à définir une correspondance entre FA $\sigma$ I et mesures dont nous montrerons bientôt le caractère canonique.

V.1. Dans [10], Meyer a montré que sous l'hypothèse $(F)$ un potentiel fini était le potentiel d'une FAN si et seulement si c'était le potentiel d'une mesure ne chargeant pas les ensembles polaires. Nous allons d'abord montrer ici, sans $(F)$, que cette mesure est alors la mesure $\nu_{A}$, introduite dans les paragraphes précédents. Le résultat de Weil [19] sur le comportement des fonctions excessives sur les trajectoires de $X$ sera essentiel pour la démonstration qui suit. A quelques détails près cette démonstration est celle de [2]; nous la reproduisons ici pour la commodité du lecteur.

Proposition. Si A est une FANoI dont le $\alpha$-potentiel est fini $\xi$-presque partout $(\alpha \geqq 0)$, on $a$

$$
E_{x} \int_{0}^{\infty} e^{-\alpha t} d A_{t}=\int U^{\alpha}(x, y) \nu_{A}(d y)
$$

Démonstration. Supposons d'abord $\alpha>0$, et $A$ intégrable, et soit $\phi$ une fonction de $b \mathscr{E}_{+} \cap \mathscr{L}^{1}(\xi)$; considérons l'expression

$$
\int \hat{U}^{\alpha} \phi(x) \nu_{A}(d x)=\lim _{\beta \rightarrow \infty} \beta E_{\xi} \int_{0}^{\infty} e^{-\beta s} \hat{U}^{\alpha} \phi\left(X_{s}\right) d A_{s} ;
$$

pour $P_{\xi}$-presque toute trajectoire l'ensemble des temps de discontinuités est dénombrable et la mesure $d A_{\mathrm{s}}$ ne charge pas ces temps, puisque $A$ est supposée naturelle. On a donc

$$
\int_{0}^{\infty} e^{-\beta s} \hat{U}^{\alpha} \phi\left(X_{s}\right) d A_{s}=\int_{0}^{\infty} e^{-\beta s} \hat{U}^{\alpha} \phi\left(X_{s^{-}}\right) d A_{s} ;
$$

or d'après [19], la fonction $t \leadsto \hat{U}^{\alpha} \phi\left(X_{t^{-}}\right)$étant continue à gauche sur $) 0, \zeta($, la suite des fonctions

$$
\sum_{k \geqq 0} \exp [-\beta k / n] 1_{) k / n,(k+1) / n)}(s) \hat{U}^{\alpha} \phi\left(X_{(k / n)-}\right)
$$


tend vers la fonction $e^{-\beta s} \hat{U}^{\alpha} \phi\left(X_{s^{-}}\right)$sur $) 0, \zeta$ ( en restant majorée par la fonction $e^{-\beta s}\|\phi\|_{\infty} / \alpha$ qui est, par hypothèse, $P_{\xi}$-presque sûrement intégrable pour le mesure $d A_{s}$; comme le mesure $d A_{s}$ ne charge que ) $0, \zeta$ (, on a $P_{\xi}$-presque sûrement

$$
\int_{0}^{\infty} e^{-\beta s} \hat{U}^{\alpha} \phi\left(X_{s^{-}}\right) d A_{s}=\lim _{n \rightarrow \infty} \sum_{k \geqq 0} \exp [-\beta k / n] \hat{U}^{\alpha} \phi\left(X_{(k / n)-}\right)\left(A_{((k+1) / n)-} A_{k / n}\right) .
$$

Posons

$$
\begin{aligned}
Y_{n} & =\sum_{k \geqq 0} \exp [-\beta k / n] \frac{\|\phi\|_{\infty}}{\alpha}\left(A_{((k+1) / n)-} A_{k / n}\right), \\
Z_{n} & =\sum_{k \geqq 0} \exp [-\beta k / n] \hat{U}^{\alpha} \phi\left(X_{(k / n)-}\right)\left(A_{((k+1) / n)-} A_{k / n}\right) .
\end{aligned}
$$

On a

$$
\lim _{n} Y_{n}=Y=\frac{\|\phi\|_{\infty}}{\alpha} \int_{0}^{\infty} e^{-\alpha t} d A_{t} P_{\xi} \text {-p.s. }
$$

et cette limite est décroissante $\left(Y_{n} \geqq Y\right)$, par suite

$$
E_{\xi}\left(Y_{n}-Y\right) \leqq \frac{\|\phi\|_{\infty}}{\alpha} \sum_{k \geqq 0} \exp [-\beta k / n](1-\exp [-\beta / n]) E_{\xi} A_{1 / n} \leqq \frac{\|\phi\|_{\infty}}{\alpha}\left\|\nu_{A}\right\| \frac{1}{n} .
$$

Il en résulte que $Y_{n} \rightarrow Y$ dans $L^{1}\left(P_{\xi}\right)$, et comme $Z_{n} \leqq Y_{n}$,

$$
Z_{n} \rightarrow \int_{0}^{\infty} e^{-\beta s} \hat{U}^{\alpha} \phi\left(X_{s^{-}}\right) d A_{s}
$$

dans $L^{\prime}\left(P_{\xi}\right)$, donc

$$
E_{\xi} \int_{0}^{\infty} e^{-\beta s} \hat{U}^{\alpha} \phi\left(X_{s}\right) d A_{s}=\lim _{n \rightarrow \infty} E_{\xi} \sum_{k \geqq 0} \exp [-\beta k / n] \hat{U}^{\alpha} \phi\left(X_{(k / n)-}\right)\left(A_{((k+1) / n)-} A_{k / n}\right) .
$$

En utilisant la propriété de Markov, le fait que $\xi$ est excessive et que $X_{k / n}=X_{(k / n)-}$ $P_{\xi}$-presque sûrement, on obtient

$$
\begin{aligned}
\int \hat{U}^{\alpha} \phi(y) \nu_{A}(d y) & =\lim _{\beta \rightarrow \infty} \lim _{n \rightarrow \infty} \beta \sum_{k \geqq 0} E_{\xi}\left(\exp [-\beta k / n] \hat{U}^{\alpha} \phi\left(X_{k / n}\right) E_{X_{k / n}}\left(A_{1 / n}\right)\right) \\
& \leqq \lim _{\beta \rightarrow \infty} \lim _{n \rightarrow \infty}\left(\beta \sum_{k \geqq 0} \exp [-\beta k / n]\right) \int \hat{U}^{\alpha} \phi(y) E_{y}\left(A_{1 / n}\right) \xi(d y) \\
& =\lim _{\beta \rightarrow \infty} \lim _{n \rightarrow \infty} \frac{\beta}{1-e^{-\beta / n}} \int \phi(y) U^{\alpha}\left(E \cdot\left(A_{1 / n}\right)\right)(y) \xi(d y) .
\end{aligned}
$$

Or pour tout $\beta$,

$$
\lim _{n \rightarrow \infty} \frac{1}{n} \frac{\beta}{1-e^{-\beta / n}}=1 \text {, }
$$


ce qui entraine

$$
\begin{aligned}
\int \hat{U}^{\alpha} \phi(y) \nu_{A}(d y) & \leqq \lim _{n \rightarrow \infty} n \int \phi(y) U^{\alpha}\left(E \cdot\left(A_{1 / n}\right)\right)(y) \xi(d y) \\
& =\lim _{n \rightarrow \infty} \int_{0}^{\infty} n e^{-\alpha t} E_{\phi \xi}\left(E_{X_{t}}\left(A_{1 / n}\right)\right) d t \\
& =\lim _{n \rightarrow \infty} \int_{0}^{\infty} n e^{-\alpha t} E_{\phi \xi}\left(A_{t+1 / n}-A_{t}\right) d t \\
& =\lim _{n \rightarrow \infty}\left\{n\left(e^{\alpha / n}-1\right) \int_{1 / n}^{\infty} e^{-\alpha s} E_{\phi \xi}\left(A_{s}\right) d s-n \int_{0}^{1 / n} e^{-\alpha s} E_{\phi \xi}\left(A_{s}\right) d s\right\} \\
& =\alpha \int_{0}^{\infty} e^{-\alpha s} E_{\phi \xi}\left(A_{s}\right) d s \\
& =\int \phi(x) U_{A}^{\alpha}(x) \xi(d x) .
\end{aligned}
$$

Nous avons donc montré que pour toute fonction de $b \mathscr{E}_{+} \cap \mathscr{L}^{1}(\xi)$

il en résulte que

$$
\int \hat{U}^{\alpha} \phi(y) \nu_{A}(d y) \leqq \int U_{A}^{\alpha}(x) \phi(x) \xi(d x)
$$

$$
\int U^{\alpha}(x, y) \nu_{A}(d y) \leqq U_{A}^{\alpha}(x) \quad \xi \text {-presque partout; }
$$

et comme les deux membres sont des fonctions $\alpha$-excessives l'inégalité a lieu partout.

Comme dans [4], nous écrirons $U^{\alpha} \nu_{A}(x)=\int U^{\alpha}(x, y) \nu_{A}(d y)$. On a

$$
U_{A}^{\alpha}-U^{\alpha} \nu_{A}=\lim _{\beta \rightarrow \infty} \beta U^{\beta+\alpha}\left(U_{A}^{\alpha}-U^{\alpha} \nu_{A}\right)=\lim _{\beta \rightarrow \infty} U^{\alpha}\left(\beta U_{A}^{\beta+\alpha}-\beta U^{\alpha+\beta} \nu_{A}\right)
$$

d'après les équations résolvantes vérifiées par $U_{A}^{\alpha}$ et $U^{\alpha} \nu_{A}$. On a donc

$$
\begin{aligned}
\int \xi(d x)\left(U_{A}^{\alpha}(x)-U^{\alpha} \nu_{A}(x)\right) & \leqq \liminf _{\beta \rightarrow \infty} \int \xi(d x) U^{\alpha}\left(\beta U_{A}^{\beta+\alpha}(x)-\beta U^{\beta+\alpha} \nu_{A}(x)\right) \\
& \leqq \frac{1}{\alpha} \liminf _{\beta \rightarrow \infty} \int \xi(d x)\left(\beta U_{A}^{\beta+\alpha}(x)-\beta U^{\beta+\alpha} \nu_{A}(x)\right) ;
\end{aligned}
$$

mais $\lim _{\beta \rightarrow \infty}\left\langle\xi, \beta U_{A}^{\beta+\alpha}\right\rangle=\left\|\nu_{A}\right\|$ et $\lim _{\beta \rightarrow \infty}\left\langle\xi, \beta U^{\beta+\alpha} \nu_{A}\right\rangle$ est égale, par dualité, à $\lim _{\beta \rightarrow \infty}\left\langle\nu_{A}, \beta \hat{U}^{\alpha+\beta} 1\right\rangle=\left\|\nu_{A}\right\|$; il en résulte que $U_{A}^{\alpha}=U^{\alpha} \nu_{A} \xi$-p.s. et par suite partout. Le cas où $\alpha=0$ et où $A$ est $\sigma$-intégrable se traitent alors aisément par des passages à la limite monotones.

V.2. Nous allons utiliser cette proposition pour approcher de la caractérisation des FAN $\sigma$ I. Soient $\tau$ et $\sigma$ deux temps d'arrêt accessibles terminaux et effilés en tout point, nous dirons qu'ils sont étrangers si $P_{x}\left(\bigcup_{k, l}\left\{\tau^{(k)}=\sigma^{(l)}\right\}\right)=0$ pour tout $x$ de $E$.

Proposition. Si $\tau$ et $\sigma$ sont étrangers et si $B^{\tau}$ et $B^{\sigma}$ sont dans $\Phi$, les mesures $\nu_{B^{\tau}}$ et $\nu_{B^{\sigma}}$ sont étrangères. 
Démonstration. Supposons d'abord qu'il existe un $\alpha>0$ tel que $U_{B}^{\alpha}$ et $U_{B}^{\alpha}$ soient finis; la borne inférieure pour l'ordre $\alpha$-fort (cf. [11]) de ces deux fonctions $\alpha$-excessives est zéro. En effet dans le cas contraire il existerait d'après les théorèmes de Meyer ([10], [4, p. 167]) une FAN $C_{t}$ non nulle telle que $B_{t}^{\tau}-C_{t}$ et $B_{t}^{\sigma}-C_{t}$ soient des FAN, or cela est impossible puisque $B^{\tau}$ et $B^{\sigma}$ ne croissent que par des sauts qui sont tous différents.

Maintenant sous les hypothèses de dualité, d'après ce que nous venons de voir

$$
U_{B^{\tau}}^{\alpha}(x)=\int U^{\alpha}(x, y) \nu_{B^{\tau}}(d y), \quad U_{B^{\sigma}}^{\alpha}(x)=\int U^{\alpha}(x, y) \nu_{B^{\sigma}}(d y)
$$

si $\nu_{B^{\tau}}$ et $\nu_{B^{\sigma}}$ ne sont pas étrangères, il existe une mesure $m$ non nulle telle que $\nu_{B^{\imath}}=m+\tilde{\nu}_{\tau}, \nu_{B^{\sigma}}=m+\tilde{\nu}_{\sigma}$; le potentiel $U^{\alpha} m$ est alors un minorant fort de $U_{B^{\tau}}^{\alpha}$ et $U_{B}^{\alpha}$, qui n'est pas nul, puisque sa nullité entrainerait $m=0$ d'après le Théorème (VI.1.15) de [4]. Cette contradiction entraine que $\nu_{B^{\tau}}$ et $\nu_{B^{\sigma}}$ sont étrangères.

Si $B^{\tau}$ et $B^{\sigma}$ appartiennent à $\Phi$, leurs traces sur les ensembles sont des FAN et d'après IV. 2 on peut choisir une suite d'ensemble $E_{n}$ de réunion $E$ tels que ces traces soient de potentiels bornés; ce qui vient d'être montré prouve que les restrictions de $\nu_{B^{\tau}}$ et $\nu_{B^{\sigma}}$ aux $E_{n}$ sont étrangères et par suite que ces mesures sont étrangères.

V.3. ThÉORÈme. Une FAN est o-intégrable si et seulement si ses sauts sont presque-sûrement finis. Il existe alors une suite d'ensembles $E_{n}$ des réunion $E$ dont, les 1-potentiels sont intégrables et bornés.

Démonstration. La nécessité a été démontrée en IV.1. Pour montrer la suffisance, on décompose la fonctionnelle $A$, d'après I.6, en la somme d'une FAC et d'une FAN purement discontinue $D_{t}$ dont les sauts sont donc p.s. finis. D'après III.1 il suffit de montrer que $D_{t}$ est $\sigma$-intégrable. En raisonnant comme en I.6 on voit que

$$
D_{t} \leqq \sum_{k=1}^{\infty} a_{k+1} \sum_{1}^{\infty} 1_{\left\{\tau_{k}^{(n)} \leqq t\right\}}=\sum_{k=1}^{\infty} D_{t}^{k}
$$

nous allons montrer qu'il existe une suite $F_{k}$ d'ensembles disjoints tels que la fonctionnelle $D_{t}^{k}$ soit portée par $F_{k}$. Si toutes les fonctionnelles $B^{\tau_{k}}$ étaient dans $\Phi$, cela résulterait de la proposition précédente, car il est clair que les sauts d'amplitudes différentes d'une fonctionnelle sont étrangers. Supposons maintenant qu'il existe deux temps $\tau_{k}$, soient $\tau_{i}$ et $\tau_{j}$, tels que $B^{\tau_{i}}$ et $B^{\tau}$, ne soient pas dans $\Phi$, il est alors clair que

$$
\lim _{n \rightarrow \infty} \tau_{i}^{(n)}=\lim _{n \rightarrow \infty} \tau_{j}^{(n)}=\inf \left\{t: A_{t}=\infty\right\}=R ;
$$

si maintenant on construit les mesures aléatoires $d B_{t}^{\tau_{i}}$ et $d B_{t}^{\tau_{j}}$ du paragraphe IV.3 associées à $\tau_{i}$ et $\tau_{j}$, les mesures vont être étrangères sur toute la droite. Leurs traces sur les ensembles construites en IV.2 sont donc deux FAN du type étudié dans la proposition précédente et par suite les mesures associées sont étrangères. 
D'après la Proposition IV.2 chaque $F_{k}$ est réunion dénombrable d'ensembles $F_{k}^{j}$ tels que $1_{F_{k}^{j}} \cdot D^{k}$ soit majorée par une fonctionnelle de 1-potentiel intégrable et borné. Si l'on pose $F=E \backslash \bigcup_{k} F_{k}$ et $E_{n}=F \bigcup_{k=1}^{n} \bigcup_{j=1}^{n} F_{k}^{j}$, les ensembles $E_{n}$ répondent à la question et $A$ est une FAN $\sigma$ I.

COROLlaire. Toutes les $F A N$ de la classe $\Phi$, toutes les $F A N$ de potentiel fini sont $\sigma$-intégrables.

Démonstration. La première partie est évidente et la deuxième résulte du fait que les FAN de potentiel fini sont dans $\Phi$.

Remarque. Les mesures $\nu_{A} \uparrow E_{n}$ sont de potentiels intégrables et bornés; cela résulte du fait que cette propriété est valable dans les cas étudiés aux paragraphes III et IV.

V.4. Sous l'hypothèse (F) de Hunt (VI.2.1. de [4]), Blumenthal et Getoor ont montré dans [6] que les deux temps d'arrêt de V.2. sont les temps d'entrée dans deux ensembles effilés. $\mathrm{Si} \tau$ et $\sigma$ sont étrangers il est clair que l'intersection de ces ensembles effilés est polaire et par suite que $\nu_{B^{\tau}}$ et $\nu_{B^{\sigma}}$ sont étrangères d'après la remarque qui termine IV.2. On peut donc se servir de cet argument pour démontrer le théorème précédent.

Nous allons ici démontrer le théorème de [6] sous nos hypothèses un peu plus faibles. Nous allons utiliser largement la démonstration de [6] mais en en supprimant l'emploi des théorèmes de théorie du potentiel.

THÉORÈME. Si $\tau$ est un temps d'arrêt accessible, terminal, effilé en tout point et tel que $P_{x}(\zeta \leqq \tau<\infty)=0$ pour tout $x$ de $E$, il existe un ensemble borélien effilé $B$ tel que $\tau=T_{B}$ presque sûrement.

Démonstration. Supposons tout d'abord que la FAN $B^{\tau}$ soit de 1-potentiel fini; pour toute fonction $f \in b \mathscr{E}_{+}$, on a alors

$$
U_{B^{\tau}}^{1} f(x)=\int U^{1}(x, y) f(y) \nu_{B^{\tau}}(d y)=\sum_{n=1}^{\infty} E_{x}\left(\exp \left[-\tau^{n}\right] f\left(X_{\tau^{n}}\right)\right)
$$

comme dans [6], on pose $\Phi(x)=E_{x}\left(e^{-\tau}\right), K_{n}=\{x: \Phi(x) \geqq 1-1 / n\}, T_{n}=T_{K_{n}}$; d'après [6], $T_{K_{n}} \uparrow \tau$ presque sûrement et $T_{n}<\tau$ sur $\{\tau<\infty\}$; comme $\tau$ est terminal, on a donc facilement $P_{K_{n}}^{1} U_{B^{\imath}}^{1} g=U_{B^{\imath}}^{1} g$, soit en utilisant la formule de dualité et le principe d'unicité des masses

$$
\hat{P}_{K_{n}}^{1} \nu_{B^{\tau}}=\nu_{B^{\tau}}
$$

La mesure $\nu_{B^{\tau}}$ est donc portée par la fermeture cofine $L_{n}$ de $K_{n}$. On pose $B=\bigcap L_{n}$; $B$ est un fermé cofin, $\nu_{B^{\tau}}$ est portée par $B$, donc $\tau \geqq T_{B}$ p.s. On montre maintenant exactement comme dans [6] que $\tau \leqq T_{B}$ p.s. ce qui démontre le résultat dans ce cas.

Si $B^{\tau}$ n'est pas de potentiel fini, il existe d'après le paragraphe IV.2. une suite $\left\{E_{k}\right\}$ d'ensembles de réunion $E$, tels que si

$$
\tau_{k}=\inf \left\{\tau^{n}: X_{\tau^{n}} \in E_{k}\right\}
$$


la fonctionnelle $B^{\tau_{k}}$ soit de 1-potentiel fini. Il existe donc un ensemble effilé $B_{k}$ contenu dans $E_{k}$ tel que $\tau_{k}=T_{B_{k}}$ p.s., on pose $B=\bigcup_{k} B_{k}$. Lorsque $k$ tend vers l'infini, $\tau_{k}$ décroit vers $\tau$ en étant égal à $\tau$ partir d'un certain rang et par suite $\tau=T_{B}$ p.s.

On remarque que $B$ n'est plus forcément un fermé cofin; mais cela est normal comme le prouve l'exemple de l'ensemble $\bigcup_{n}\{-1 / n\}$ pour la translation uniforme sur la droite. Le résultat du paragraphe suivant montre que la première partie de cette démonstration peut se faire si $B^{\tau}$ est dans $\Phi$; dans ce cas $B$ est un fermé cofin.

V.5. Nous allons maintenant réénoncer la Proposition V.1., mais nous introduisons au préalable quelques notations. Si $B$ est un ensemble presque borélien on pose

$$
V_{B}^{\alpha}(x, y)=U^{\alpha}(x, y)-P_{B}^{\alpha} U^{\alpha}(x, y)=V^{\alpha}(x, y)-U^{\alpha} \hat{P}_{B}^{\alpha}(x, y)
$$

et $V_{B}(x, y)=\lim _{\alpha \rightarrow 0} V^{\alpha}(x, y) ; V_{B}^{\alpha}(x, y)$ peut être considéré comme le noyau associé aux processus $\left(X, T_{B}\right)$ et $\left(\hat{X}, T_{B}\right)$ bien que ces processus ne soient pas forcément standards, ni définis sur le même sous-ensemble de $E$. On a alors.

THÉORÈME. Si A est une $F A N$ de $\Phi$, pour toute fonction de $\mathscr{E}_{+}$et tout $\alpha \geqq 0$,

$$
U_{A}^{\alpha} f(x)=\int U^{\alpha}(x, y) f(y) \nu_{A}(d y)
$$

pour tout ensemble presque borélien $B$

$$
E_{x} \int_{0}^{T_{B}} e^{-\alpha t} f\left(X_{t}\right) d A_{t}=\int_{E} V_{B}^{\alpha}(x, y) f(y) \nu_{A}(d y) .
$$

Démonstration. Pour démontrer la première formule, on applique la Proposition V.1. avec $f \in b \mathscr{E}_{+}$et à supports dans les ensembles sur lesquels la trace de $A$ est intégrable, puis on fait des passages à la limite croissant. Pour la deuxième on écrit

$$
\begin{aligned}
E_{x} \int_{\left[0, T_{B}[\right.} e^{-\alpha t} f\left(X_{t}\right) d A_{t} & =E_{x} \int_{0}^{\infty} e^{-\alpha t} f\left(X_{t}\right) d A_{t}-E_{x} \int_{\left[T_{B}, \infty[\right.} e^{-\alpha t} f\left(X_{t}\right) d A_{t} \\
& =U_{A}^{\alpha} f(x)-P_{B}^{\alpha} U_{A}^{\alpha} f(x) \\
& =\int\left(U^{\alpha}(x, y)-P_{B}^{\alpha} U^{\alpha}(x, y)\right) f(y) \nu_{A}(d y) \\
& =\int V_{B}^{\alpha}(x, y) \nu_{A}(d y) .
\end{aligned}
$$

Remarquons que cette formule obtenue par Blumenthal et Getoor pour les FAC "smooth" est fondamentale pour leur étude.

V.6. Appliqué aux fonctionnelles de potentiel fini, le théorème précédent et la Proposition (VI.1.15) de [4] entrainent de manière évidente-mais sans hypothèse de forte fellerénéité des résolvantes-le Théorème (VI.3.1) de [4], c'est à dire que 
si $U \mu=U_{A}<\infty$, alors pour toute $f \in \mathscr{E}_{+}^{*}$, on a $U(f \mu)=U_{A} f$. En répétant mot à mot les démonstrations de [4] on obtient sans hypothèse de forte fellerénéité les théorèmes de Meyer.

1er THÉORÈME. U $\mu$ est un potentiel naturel si et seulement si $U_{\mu}<\infty$ et $\mu$ ne charge aucun ensemble polaire.

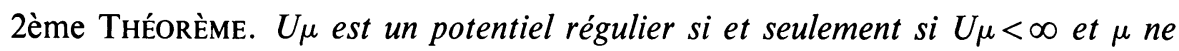
charge aucun ensemble semi-polaire.

V.7. Comme toute mesure $\sigma$-finie peut être décomposée en une mesure ne chargeant pas les semi-polaires et une mesure portée par un semi-polaire (cf. [14]) on déduit de ce qui précède une proposition énoncée dans [6] pour les FAN de potentiel fini.

Proposition. Si A est une $F A N$ de $\Phi$, il existe un ensemble semi-polaire $B$ tel que les parties continues et purement discontinues de A s'écrivent

$$
C_{t}=\int_{0}^{t} 1_{E \backslash B}\left(X_{s}\right) d A_{s} \text { et } \quad D_{t}=\int_{0}^{t} 1_{B}\left(X_{s}\right) d A_{s}
$$

Remarquons encore que si $A$ est portée par un ensemble effilé dont le temps d'entrée est $\tau$, on a $\nu_{A} \ll \nu_{B^{\tau}}$; par suite on peut étendre à toutes les FAN de $\Phi$ les représentations données dans [6]. On retrouve également (cf. [4, p. 293]) que si les semi-polaires sont polaires les fonctionnelles naturelles finies sont continues.

VI. Sur les mesures associees aux fonctionnelles additives. Les mesures $\nu_{A}$ ne chargent pas les ensembles polaires, mais il est facile de voir que toute mesure $\sigma$-finie ne chargeant pas les ensembles polaires n'est pas associée à une FAN $\sigma$; il suffit pour s'en convaincre de considérer le processus de translation uniforme sur la droite et la mesure $f \mu$ où $f=0$ sur $]-\infty, 0], f(x)=1 / x$ sur $] 0, \infty[$ et $\mu$ est la mesure de Lebesgue, où encore pour le même processus la mesure $\sum_{n=1}^{\infty} \varepsilon_{\{-1 \text { in }\}}+\varepsilon_{\{0\}}$. C'est donc un problème naturel de chercher à quelles conditions une mesure $\sigma$-finie ne chargeant pas les polaires est associée à une FANoI. On sait qu'une telle mesure peut se décomposer en la somme d'une mesure ne chargeant pas les semi-polaires et d'une mesure portée par un semi-polaire (cf. [14]). Nous pouvons donc étudier ces deux types séparément; les théorèmes V.6 montrent que le premier type ne peut être associé qu'à une FAC et le second à une FAN purement discontinue; nous traiterons ici uniquement le premier cas qui conduit aux applications du paragraphe VII. Les hypothèses sont toujours celles du début du paragraphe $\mathrm{V}$.

VI.1. Le Théorème III.1 indique des conditions nécessaires pour qu'une mesure $\sigma$-finie ne chargeant pas les semi-polaires soit associée à une FAC. Montrons qu'elles sont aussi suffisantes. 
THÉORÈME. Une mesure $v$-finie est associée à une FAC de la classe $\Phi_{c}$ si et seulement si elle vérifie les conditions suivantes:

(i) $\nu$ ne charge pas les ensembles semi-polaires.

(ii) E est réunion c'ıne suite ' $\left.E_{n}\right\}$ d'ensembles boréliens tels que

(a) $v\left(E_{n}\right)<\infty$ pour tout $n$;

(b) $\int_{E_{n}} U^{1}(x, y) \nu(d y)$ est borné en $x$ pour tout $n$;

(c) si $T_{n}=T_{E_{n}^{c}}$, et $T=\lim _{n} T_{n}$ on a $P_{x}(T \geqq \zeta)=1$ pour tout $x$ de $E$.

Démonstration. La nécessité résulte de la Proposition V.1. et du Théorème III.1. Inversement si $\nu$ vérifie toutes ces conditions, il existe pour chaque $n$, d'après le 2ème Théorème V.6, une FAC $A^{n}$ telle que

$$
U_{A^{1}}^{1}(x)=\int_{E_{n}} U^{1}(x, y) \nu(d y)
$$

et l'on a $\nu_{A^{n}}(f)=\lim _{\alpha \rightarrow \infty} \int_{E_{n}} \alpha \hat{U}^{\alpha}(y) f(y) \nu(d y)=\nu\left(1_{E_{n}} f\right)$, soit $\nu_{A^{n}}=\nu \uparrow E_{n}$. On voit donc que $A^{n}$ est intégrable, donc de la classe $\Phi_{c}$ et d'après l'unicité de la correspondance si $m>n$ on a $A^{n}=1_{E_{n}} A_{m}$. Posons $A_{t}=\lim _{n \rightarrow \infty} A_{t}^{n}$; comme $A_{T_{n} \wedge t}^{m}=A_{T_{n} \wedge t}^{n}$ pour tout $m>n$, il est clair que $A_{T_{n} \wedge t}$ est fini presque-sûrement et par suite que $A_{0}=0$ p.s. et que $A_{t}$ est finie p.s. sur [0,T[; il résulte alors de la Proposition I.5. que $A_{t}$ est une FAC de $\Phi_{c}$. Il reste à montrer que $\nu_{A}=\nu$.

Appelons $V_{n}^{\alpha}(x, y)$ le noyau défini en V.5 associé à $E_{n}^{c}$, et soit $f \in b \mathscr{E}_{+}$à support dans l'intérieur fin $E_{n}^{f}$ de $E_{n}$, comme $A_{T_{n} \wedge t}=A_{T_{n} \wedge t}^{n}$ p.s., on a pour tout $x$ de $E$

$$
E_{x} \int_{0}^{T_{n}} e^{-\alpha t} f\left(X_{t}\right) d A_{t}=E_{x} \int_{0}^{T_{n}} e^{-\alpha t} f\left(X_{t}\right) d A_{t}^{n}
$$

soit d'après la Proposition V.5

$$
\int V_{n}^{\alpha}(x, y) f(y) \nu_{A}(d y)=\int V_{n}^{\alpha}(x, y) f(y) \nu(d y)
$$

En intégrant par rapport à $\xi$ et en multipliant par $\alpha$ on obtient

$$
\int_{E} \alpha \hat{V}_{n}^{\alpha}(E, y) f(y) \nu_{A}(d y)=\int_{E} \alpha \hat{V}_{n}^{\alpha}(E, y) f(y) \nu(d y)
$$

où $V_{n}^{\alpha}(\cdot, y)$ est la résolvante du processus $\left(\hat{X}, T_{n}\right)$; mais pour tous les points $y$ de $E \backslash\left(\hat{E}_{n}^{c}\right)_{r}$, l'expression $\alpha \hat{V}_{n}^{\alpha}(E, y)$ tend vers 1 lorsque $\alpha$ tend vers l'infini, en restant borné par 1, et cet ensemble ne diffère de $E \backslash\left(E_{n}^{c}\right)_{r}$ que par un ensemble semipolaire $\left[4\right.$, p. 264] donc de mesure nulle pour $v$ et $\nu_{A}$; on peut donc passer à la limite pour obtenir

$$
\int_{E} f(y) \nu_{A}(d y)=\int_{E} f(y) \nu(d y)
$$

il en résulte que $\nu_{A}$ coïncide avec $\nu$ sur $\stackrel{\circ}{E}_{n}^{f}$; comme il résulte des hypothèses que $E=\bigcup_{n} \stackrel{\circ}{E}_{n}^{f}$, on a $\nu_{A}=\nu$. 
Remarque. Si un point $x_{0}$ est régulier pour lui même la fonction $x \rightarrow U^{\alpha}\left(x, x_{0}\right)$ est bornée. En effet, il existe dans ce cas [4, V.3. 13] un temps local en $x_{0}$, c'est à dire que la masse de Dirac en $x_{0}$ vérifie les hypothèses du théorème précédent. Ceci est à rapprocher de la Proposition VI.4.11 de [4].

VI.2. La même démonstration nous donne sans changement, en tenant compte de la Remarque 2 à la fin de V.3 et en utilisant plus pleinement la Proposition I.5, le résultat suivant.

Proposition. Une mesure $\nu$-finie ne chargeant pas les semi-polaires est associée à une $F A C$ si et seulement si il existe une suite $E_{n}$ d'ensembles de réunion $E$ tels que

(1) $\nu\left(E_{n}\right)<\infty$ pour tout $n$,

(2) $\int_{E_{n}} U^{1}(x, y) \nu(d y)$ est borné en $x$ pour tout $n$,

(3) $T=\lim T_{E_{n}^{c}}$ est positif p.s.

VI.3. Dans les hypothèses des théorèmes précédents la condition essentielle est celle qui porte sur les temps d'entrée, comme le montre la proposition suivante.

Proposition. Pour toute mesure $\nu$-finie ne chargeant pas les ensembles semipolaires il existe une suite croissante d'ensembles boréliens $\left\{E_{n}\right\}$ de réunion $E$ tels que pour tout $n$ : (1) $\nu\left(E_{n}\right)<\infty$; (2) $\int_{E_{n}} U^{1}(x, y) \nu(d y)$ soit borné en $x$.

Démonstration. Nous ne la faisons que sommairement car elle est pour l'essentiel dans [14]. On part d'une suite $\left\{F_{n}\right\}$ telle que $\nu\left(F_{n}\right)<\infty$, pour tout $n$; il en résulte que

$$
g_{n}(x)=\int_{F_{n}} U^{1}(x, y) \nu(d y)
$$

est intégrable donc fini sauf sur un ensemble polaire $P$. On pose $G_{n}^{k}=\left\{x: g_{n}(x) \leqq k\right\}$, puis $H_{n}^{k}=F_{n} \cap G_{n}^{k}$ et l'on obtient aisément que

$$
\int_{H_{n}^{k}} U^{1}(x, y) \nu(d y) \leqq k
$$

on pose alors $E_{n}=\bigcup_{m=1}^{n} \bigcup_{k=1}^{n} H_{m}^{k} \cup\left(F_{n} \cap P\right)$; les $E_{n}$ répondent àl a question et nous remarquerons que $E_{n} \subset F_{n}$ pour chaque $n$.

VII. Dualité et changement de temps. Comme dans [5], il est naturel de se demander si une mesure associée à une $\mathrm{FAC}$ du processus $X$ est aussi associée à une FAC du processus $\hat{X}$; l'exemple du début du paragraphe VI montre qu'il n'en est rien. Cependant en se restreignant aux fonctionnelles de $\Phi_{c}$ on peut obtenir le résultat que Blumenthal et Getoor ont obtenu pour les fonctionnelles "smooth".

VII.1. THÉORÈME. Si v est associée à une fonctionnelle de la classe $\Phi_{c}$ il existe un ensemble polaire $P$ et une fonctionnelle $\hat{A} d u$ processus $\hat{X}$ restreint $\grave{a} E \backslash P$, qui appartient $\grave{a} \Phi_{c}$ et qui est associée à $\nu$. 
Démonstration. Si $\nu$ est associée à une FAC de $\Phi_{c}$, nous avons vu qu'il existe une suite $\left\{E_{n}\right\}$ d'ensembles boréliens de réunion $E$ tels que

(1) $\nu\left(E_{n}\right)<\infty$,

(2) $\int_{E_{n}} U^{1}(x, y) \nu(d y)$ est borné en $x$,

(3) si $T_{n}=T_{E_{n}^{c}}$ et $T=\lim T_{n}$, on a $P_{x}(T=\zeta)=1$

pour tout $x$ de $E$. Comme $\nu\left(E_{n}\right)<\infty$ les expressions

$$
\int_{E_{n}} \nu(d x) U^{1}(x, y)
$$

sont $\xi$-intégrables et par suite finies sauf sur un ensemble polaire $Q_{n}$, si nous posons $Q=\cup_{n} Q_{n}, Q$ est un ensemble polaire que nous pouvons retirer de l'espace sans rien charger par ailleurs. Nous aurons donc une suite $\left\{E_{n}\right\}$ d'ensembles de réunion l'espace entier et ayant en outre la propriété que les 1-copotentiels de $\nu$ restreinte à $E_{n}$ sont partout finis.

Soit maintenant $A \in \mathscr{E}$ et $\xi(A)<\infty$, la condition (3) entraine que

$$
\int_{A} \xi(d x) P_{T_{n}}^{1} U^{1}(x, E)=\int_{A} \xi(d x) E_{x} \int_{T_{n}}^{\zeta} e^{-t} 1_{E}\left(X_{t}\right) d t \underset{n \rightarrow \infty}{\longrightarrow} 0,
$$

soit en appliquant la formule de dualité

$$
\int \hat{U}^{1}(A, y) \hat{P}_{T_{n}}^{1}(d y, z) \xi(d z) \underset{n \rightarrow \infty}{\longrightarrow} 0 ;
$$

on a donc finalement

$$
\hat{E}_{\xi} \int_{T}^{\zeta} e^{-t} 1_{A}\left(X_{t}\right) d t=0,
$$

et en faisant croitre $A$ vers $E$

$$
\hat{E}_{\xi} \int_{T}^{\zeta} e^{-t} 1_{E}\left(X_{t}\right) d t=0
$$

soit $T \geqq \zeta \hat{\boldsymbol{P}}_{\xi}$-p.s. Si $N$ est l'ensemble des $x$ tels que $\hat{P}_{x}(T<\zeta)>0$ on a donc $\xi(N)=0$; nous pouvons maintenant reprendre le raisonnement du Théorème VI.1. Il existe une suite $\hat{A}_{t}^{n}$ de FACI associées aux $\nu\left\lceil E_{n}\right.$, et l'on pose $\hat{A}_{t}=\lim \hat{A}_{t}^{n} ; \hat{A}_{t}$ est continue et finie sur [0, $\zeta\left[\hat{P}_{x}\right.$-p.s. pour tout $x \notin N$, et d'après la Proposition I.5 si $\hat{A}_{t}$ n'est pas une FAC, l'ensemble $R=\left\{x: \hat{P}_{x}\left(\hat{A}_{0+}=\infty\right)=1\right\}$ n'est pas vide, et $R \subset N$. Même si $\hat{A}_{t}$ n'est pas une FAC, elle vérifie d'après I.5 la propriété d'additivité forte et par suite si $K$ est un compact contenu dans $R, A_{T_{K+}}=\infty \operatorname{sur}\left(T_{K}<\zeta\right) \hat{P}_{x}$-p.s. pour tout $x$ de $E$; il en résulte que pour tout $x \notin N P_{x}\left(T_{K}<\zeta\right)=0$.

Nous copions maintenant le raisonnement de Blumenthal et Getoor [5] pour montrer que $R$ est polaire. Soit $x \in E$, comme $\xi(N)=0$ il est possible de trouver une suite $\left\{t_{j}\right\}$ tendant vers zéro et telle que $\hat{P}_{x}\left(X_{t} \in N\right)=0$ pour tout $j$. Maintenant

$$
\hat{P}_{x}\left(t_{j}+T_{K} \circ \theta_{j}<\zeta\right) \leqq \hat{E}_{x}\left(1_{\left\{t_{j}<\zeta\right\}} \hat{E}_{X_{t_{j}}}\left(T_{K}<\zeta\right)\right)=0 ;
$$

en faisant tendre $t_{j}$ vers zéro $\hat{P}_{x}\left(T_{K}<\zeta\right)=0$; par suite tout compact contenu dans $R$ est polaire et $R$ est polaire. On pose $P=Q \cup R$. 
Il est clair que $\hat{A}_{t}$ est une FAC du processus $\hat{X}$ restreint à $E \backslash P$, et comme $\hat{A}_{t}$ est alors finie $\hat{P}_{\xi}$-p.s. sur [0, $\zeta\left[\right.$ elle est finie $P_{x}$-p.s. pour tout $x$ de $E \backslash N$ d'après le raisonnement fait en II.3. Il ne reste plus qu'à montrer que $\hat{A}_{t}$ est associée à $\nu$ ce qui ce fait comme en VI.1.

Remarque. Dans [5] Blumenthal et Getoor donnent un exemple montrant que l'ensemble polaire exceptionnel ne peut être éliminé.

VII.2. Aux fonctionnelles $A_{t}$ et $\hat{A}_{t}$ du paragraphe précédent, nous associons deux familles de noyaux, en posant

et

$$
W_{\beta A}^{\alpha} f(x)=E_{x} \int_{0}^{\infty} \exp \left[-\beta A_{t}\right] e^{-\alpha t} f\left(X_{t}\right) d t, \quad x \in E,
$$

$$
\hat{W}_{\beta A}^{\alpha} f(x)=\hat{E}_{x} \int_{0}^{\infty} \exp \left[-\beta \hat{A}_{t}\right] e^{-\alpha t} f\left(X_{t}\right) d t, \quad x \in E \backslash P ;
$$

ce sont les résolvantes associées aux fonctionnelles multiplicatives $\exp \left[-\beta A_{t}\right]$ et $\exp \left[-\beta \hat{A}_{t}\right]$. On posera d'autre part

$$
\begin{aligned}
& V_{\beta A}^{\alpha} f(x)=E_{x} \int_{0}^{\infty} \exp \left[-\beta A_{t}\right] e^{-\alpha t} f\left(X_{t}\right) d A_{t}, \\
& \hat{V}_{\beta A}^{\alpha} f(x)=\hat{E}_{x} \int_{0}^{\infty} \exp \left[-\beta \hat{A}_{t}\right] e^{-\alpha t} f\left(X_{t}\right) d \hat{A}_{t} ;
\end{aligned}
$$

pour $\alpha=0$, on écrira simplement $V_{\beta A}$ (resp. $\hat{V}_{\beta A}$ ) et l'on obtient ainsi les résolvantes des processus obtenus par changement de temps à partir de $A_{t}$ (resp. $\hat{A}_{t}$ ). Notre but est de montrer comme dans [5] et [16] que les noyaux $W_{\beta A}^{\alpha}\left(\right.$ resp $\left.V_{\beta A}^{\alpha}\right)$ sont en dualité avec $\hat{W}_{\beta A}^{\alpha}\left(\operatorname{resp} \hat{V}_{\beta A}^{\alpha}\right)$. Des calculs simples, du type de celui effectué en III.1, montrent que ces noyaux vérifient les relations suivantes (cf. [4, p. 160] et [16]):

$$
\begin{aligned}
U_{A}^{\alpha} V_{\beta A}^{\alpha} & =V_{\beta A}^{\alpha} U_{A}^{\alpha}=\left(U_{A}^{\alpha}-V_{\beta A}^{\alpha}\right) / \beta, \\
U_{A}^{\alpha} W_{\beta A}^{\alpha} & =\left(U^{\alpha}-W_{\beta A}^{\alpha}\right) / \beta=V_{\beta A}^{\alpha} U^{\alpha} .
\end{aligned}
$$

On a d'autre part les équations résolvantes

et

$$
W_{\beta A}^{\lambda}-W_{\beta A}^{\mu}+(\lambda-\mu) W_{\beta A}^{\lambda} W_{\beta A}^{\mu}=0,
$$

$$
V_{\lambda A}-V_{\mu A}+(\lambda-\mu) V_{\lambda A} V_{\mu A}=0 \text {. }
$$

Dans les développements qui suivent nous ferons, afin d'alléger l'exposé, comme si $P$ était vide; $P$ étant de mesure nulle pour $\xi$ et $\nu$, le fait de tenir compte de $P$ n'introduirait aucune difficulté supplémentaire.

Il est facile de voir que les noyaux $W$ et $\hat{W}$ (resp $V$ et $\hat{V}$ ) sont absolument continus par rapport à $\xi$ (resp $\nu$ ) et il ressort immédiatement des équations (1) et (2) que si $g_{\beta}^{\alpha}(x, y)$ est une densité de $W_{\beta A}^{\alpha}$ par rapport à $\xi$, c'est aussi une densité de $V_{\beta A}^{\alpha}$ par rapport à $\nu$. Il est clair que l'on obtient une telle densité en posant:

$$
g_{\beta}^{\alpha}(x, y)=U^{\alpha}(x, y)-\beta \int V_{\beta A}^{\alpha}(x, d z) U^{\alpha}(z, y)
$$


on posera de même

$$
\hat{g}_{\beta}^{\alpha}(x, y)=U^{\alpha}(x, y)-\beta \int U^{\alpha}(x, z) \hat{V}_{\beta A}^{\alpha}(d z, y) .
$$

Nous allons maintenant nous inspirer de Nagasawa et Sato [16]. On suppose d'abord que $\nu$ est de $\alpha$-potentiel et de $\alpha$-co-potentiel borné; soient $f, g \in b \mathscr{E}_{+} \cap \mathscr{L}^{1}(\xi)$, on $\mathrm{a}$ :

$$
\int g_{\beta}^{\alpha}(x, y) g(y) d y=\int U^{\alpha}(x, y) g(y) d y-\beta \iint g_{\beta}^{\alpha}(x, z) \nu(d z) U^{\alpha}(z, y) g(y) d y ;
$$

mais on peut intégrer ceci par rapport à $f \xi$ pour obtenir

$$
\begin{aligned}
& \iint f(x) d x g_{\beta}^{\alpha}(x, y) g(y) d y \\
& \quad=\iint f(x) d x U^{\alpha}(x, y) g(y) d y-\beta \iiint f(x) d x g_{\beta}^{\alpha}(x, z) \nu(d z) U^{\alpha}(z, y) g(y) d y
\end{aligned}
$$

comme ceci est vrai quelque soit $g$, cela entraine que $\xi$-p.s. en $y$

$$
\int f(x) d x g_{\beta}^{\alpha}(x, y)=\int f(x) d x U^{\alpha}(x, y)-\beta \iint f(x) d x g_{\beta}^{\alpha}(x, z) \nu(d z) U^{\alpha}(z, y) .
$$

D'autre part en appliquant la relation $\hat{W}_{\beta A}^{\alpha}=\hat{U}^{\alpha}-\beta \hat{U}_{A}^{\alpha} \hat{W}_{\beta A}^{\alpha}$, on a par définition de $\hat{g}_{\beta}^{\alpha}$,

$$
\int f(x) d x \hat{g}_{\beta}^{\alpha}(x, y)=\int f(x) d x U^{\alpha}(x, y)-\beta \iint f(x) d x \hat{g}_{\beta}^{\alpha}(x, z) \nu(d z) U^{\alpha}(z, y) .
$$

En comparant ces deux expressions, il vient

$$
\int f(x) d x\left(g_{\beta}^{\alpha}(x, y)-\hat{g}_{\beta}^{\alpha}(x, y)\right)=-\beta \iint f(x) d x\left(g_{\beta}^{\alpha}(x, z)-\hat{g}_{\beta}^{\alpha}(x, z)\right) \nu(d z) U^{\alpha}(z, y) .
$$

Posons $h(y)=\int f(x) d x\left(g_{\beta}^{\alpha}(x, y)-\hat{g}(x, y)\right) ; h$ est une fonction bornée presque sûrement car $g_{\beta}^{\alpha}(x, y) \leqq U^{\alpha}(x, y)$ et $\hat{g}_{\beta}^{\alpha}(x, y) \leqq U^{\alpha}(x, y)$ et à cause de l'hypothèse faite sur $\nu$, et l'on a

$$
h(y)=-\beta \int h(x) \hat{U}_{A}^{\alpha}(d x, y)=-\beta \hat{U}_{A}^{\alpha} h(y) .
$$

Soit $M<\infty$ une borne supérieure de $\hat{U}_{A}^{\alpha}(x, E)$, pour $\beta<M$, on a $\|h\|_{\infty}<k\|h\|_{\infty}$ avec $k<1$ donc $h=0 \xi$-p.s. Ceci entraine qu'il existe donc un nombre $\beta_{0}>0$ tel que pour tout $\beta \leqq \beta_{0}$ on ait

$$
\int f(x) W_{\beta A}^{\alpha} g(x) d x=\int \hat{W}_{\beta A}^{\alpha} f(x) \cdot g(x) d x .
$$

Un calcul simple montre que

$$
W_{\beta_{0} A}^{\alpha}-W_{\beta A}^{\alpha}=\left(\beta-\beta_{0}\right) W_{\beta_{0} A}^{\alpha} W_{\beta A}^{\alpha} ;
$$

le raisonnement que nous venons de faire avec l'équation (2) nous pouvons le 
refaire avec cette nouvelle équation où cette fois $W_{\beta_{0} A}^{\alpha}$ va jouer le rôle tenu précédement par $U_{A}^{\alpha}$, c'est à dire qu'on aura

$$
g_{\beta}^{\alpha}(x, y)=g_{\beta_{0}}^{\alpha}(x, y)-\left(\beta-\beta_{0}\right) \int g_{\beta}^{\alpha}(x, z) \nu(d z) g_{\beta_{0}}^{\alpha}(z, y),
$$

et les relations analogues. On trouvera que la relation (3) est encore valable pour l'intervalle $\left(0,2 \beta_{0}\right)$ et par suite sur toute la droite.

Maintenant la mesure $\nu$ qui nous intéresse est limite croissante de mesures associées à des fonctionnelles et dont les $\alpha$ - (co-) potentiels sont bornés; un passage à la limite évident entraine alors que pour tout $\alpha, \beta \geqq 0$, et $f, g \in b \mathscr{E}_{+}$

$$
\int f(x) W_{\beta A}^{\alpha} g(x) d x=\int \hat{W}_{\beta A}^{\alpha} f(x) g(x) d x .
$$

Nous pouvons maintenant énoncer le

THÉORÈmE. Il existe des noyaux fonctions $U_{\beta}^{\alpha}(x, y)$ jouissant des propriétés suivantes:

(1) les fonctions $x \rightarrow U_{\beta}^{\alpha}(x, y)$ sont finement continues et $W_{\beta A}^{\alpha}$-excessives pour tout $y$.

(2) les fonctions $y \rightarrow U_{\beta}^{\alpha}(x, y)$ sont cofinement continues et $\hat{W}_{\beta A^{-}}^{\alpha}$-excessives sur $E \backslash P$.

(3) ce sont des densités pour les noyaux $W_{\beta A}^{\alpha}, \hat{W}_{\beta A}^{\alpha}, V_{\beta A}^{\alpha}, \hat{V}_{\beta A}^{\alpha}$ par rapport aux mesures $\xi$ et $\nu$.

Enfin on a entre les divers noyaux les relations suivantes où $f, g \in b \mathscr{E}_{+}$

$$
\begin{aligned}
\int_{E} W_{\beta A}^{\alpha} f \cdot g d \xi & =\int_{E \backslash P} f \cdot \hat{W}_{\beta A}^{\alpha} g d \xi, \\
\int_{E} V_{\beta A}^{\alpha} f \cdot g d \nu & =\int_{E \backslash P} f \cdot \hat{V}_{\beta A}^{\alpha} g d \nu, \\
\int_{E} V_{\beta A}^{\alpha} f \cdot g d \xi & =\int_{E \backslash P} f \cdot \hat{W}_{\beta A}^{\alpha} g d \nu .
\end{aligned}
$$

Démonstration. La relation (i) vient d'être démontrée, l'existence des noyaux fonctions $U_{\beta}^{\alpha}(x, y)$ résulte du théorème de Kunita et Watanabe $[4$, p. 254]. Le reste découle alors facilement des relations (1) et (2).

Remarque. (1) Pour $\beta=0, U_{0}^{\alpha}(x, y)=U^{\alpha}(x, y)$.

(2) Les intégrales au second membre peuvent s'étendre à $E$ tout entier puisque $\xi$ et $\nu$ ne chargent pas $P$.

(3) Par des arguments de continuité fine on peut montrer que $U_{\beta}^{0}(x, y)$ est $\beta$-excessive pour $V_{\beta A}$.

VII.3. Si les processus qui viennent d'être étudiés étaient standards, les résultats précédents montreraient qu'ils sont en dualité au sens du paragraphe V. Sur la vue du Corollaire III.3.16 de [4] on peut donc énoncer 
THÉORÈME 1. Si $A_{t}$ et $\hat{A}_{t}$ sont $\mathscr{F}_{t}^{0}$-mesurables les sous-processus associés sont en dualité par rapport $\grave{a} \xi$.

Remarquons que ceci est le cas si $A_{t}=\int_{0}^{t} f\left(X_{s}\right) d s$ où $f \in b \mathscr{E}_{+}$; on retrouve ainsi un résultat de Hunt [9, partie III].

D'après $[4$, p. 233] on a donc aussi

THÉORÈME 2. Si les supports de $A_{t}$ et $\hat{A}_{t}$ sont fermés dans $E$ (en particulier si $\nu$ charge tous les ouverts fins) les processus $X_{\tau_{t}}$ et $\hat{X}_{\tau_{t}}$ obtenus par les changements de temps associés à $A_{t}$ et $\hat{A}_{t}$ sont en dualité par rapport à $\nu$.

Ce théorème contient les résultats de [5].

\section{REFERENCES}

1. J. Azéma, M. Kaplan-Duflo and D. Revuz, Mesure invariante sur les classes récurrentes des processus de Markov, Z. Wahrscheinlichkeitstheorie und Verw. Gebiete 8 (1967), 157-181. MR 36 \#6005.

2. - - Propriétés relatives des processus de Markov récurrents, $\mathrm{Z}$. Wahrscheinlichkeitstheorie und Verw. Gebiete 13 (1969), 286-314.

3. - _ Récurrence fine des processus de Markov, Ann. Inst. Henri Poincaré Sect. B. 2 (1965/66), 185-220. MR 33 \#8029.

4. R. M. Blumenthal and R. K. Getoor, Markov processes and potential theory, Academic Press, New York, 1968.

5. - Additive functionals of Markov processes in duality, Trans. Amer. Math. Soc. 112 (1964), 131-163. MR 28 \#3483.

6. - Accessible terminal times, Proc. Fifth Berkeley Sympos. Math. Statist. and Prob. (Berkeley, Calif., 1965/66), vol. II: Contributions to Probability Theory, part II, Univ. of California Press, Berkeley, Calif., 1967, pp. 1-8. MR 35 \#3748.

7. C. Doleans, Fonctionnelles additives parfaites, Séminaire de Calcul des Probabilités, vol. II, Springer, Berlin, 1968.

8. H. P. McKean and H. Tanaka, Additive functionals of the Brownian path, Mem. Coll. Sci. Univ. Kyoto Ser. A Math. 33 (1960/61), 479-506. MR 24 \#A1147.

9. G. A. Hunt, Markoff processes and potentials. I, II, III, Illinois J. Math. 1 (1957), 44-93; 316-369; ibid. 2 (1958), 151-213. MR 19, 951; MR 21 \#5824.

10. P.-A. Meyer, Fonctionnelles multiplicatives et additives de Markov, Ann. Inst. Fourier (Grenoble) 12 (1962), 125-230. MR 25 \#3570.

11. _- Processus de Markov, Lecture Notes in Math., no. 26, Springer-Verlag, Berlin, 1967. MR 36 \#2219.

12. —_, Intégrales stochastiques. I, II, III, IV, Séminaire de Probabilités (Univ. Strasbourg, 1966/67), vol. I, Springer, Berlin, 1967, pp. 72-162. MR 37 \#7000.

13. - Un lemme de théorie des martingales, Séminaire de calcul des probabilités, vol. III, Springer, Berlin, 1969.

14. —_ Un résultat de théorie du potentiel, Séminaire de calcul des probabilités, vol. III, Springer, Berlin, 1969.

15. —_, Probabilités et potentiels, Actualités Sci. Indust., no. 1318, Hermann, Paris, 1966. MR 34 \#5118.

16. M. Nagasawa and K. Sato, Some theorems on time change and killing of Markov processes, Kōdai Math. Sem. Rep. 15 (1963), 195-219. MR 29 \#1669.

17. V. A. Volkonskiǐ, Random substitution of time changes in strong Markov processes, Teor. Verojatnost. i Primenen. 3 (1958), 332-350. (Russian) MR 20 \#7344. 
18. V. A. Volkonskil, Additive functionals of Markov processes, Trudy Moskov. Mat. Obšc. (1960), 9 143-189; English transl., Selected Transl. Math. Stat. and Prob., vol. 5, Amer. Math. Soc., Providence, R. I., 1965. MR 25 \#610.

19. M. Weil, Propriétés de continuité fine des fonctions co-excessives, Z. Wahrscheinlichkeitstheorie und Verw. Gebiete 12 (1969), 75-86.

20. A. D. Ventcel', Non-negative additive functionals of Markov processes, Dokl. Akad Nauk SSSR 137 (1961), 17-20=Soviet Math. Dokl. 2 (1961), 218-221. MR 22 \#10018.

21. D. V. Widder, The Laplace transform, Princeton Math. Series, vol. 6, Princeton Univ. Press, Princeton, N. J., 1941. MR 3, 232.

\section{Laboratoire de Calcul des Probabilités, Paris, France}

